Pacific Journal of Mathematics

EXTREME EIGEN VALUES OF TOEPLITZ FORMS
ASSOCIATED WITH JACOBI POLYNOMIALS 


\section{EXTREME EIGEN VALUES OF TOEPLITZ FORMS ASSOCIATED WITH JACOBI POLYNOMIALS}

\section{I. HIRSCHMAN, JR.}

Introduction. Let $t(\theta)$ be a real function in $L^{1}(T)$ where $T$ is the real numbers modulo 1 , and let

$$
\begin{aligned}
c(k) & =\int_{T} t(\theta) e^{-2 \pi i k \theta} d \theta \quad k=0,1, \cdots, \\
C_{n} & =[c(j-k)]_{j, k=0, \cdots, n} .
\end{aligned}
$$

$C_{n}$ is the Toeplitz matrix of index $n$ associated with $t(\theta) . C_{n}$ is clearly Hermitian and thus has real eigen values,

$$
\lambda_{n, 1} \geqq \lambda_{n, 2} \geqq \cdots \geqq \lambda_{n, n+1} \text {. }
$$

For some time studies have been made of the asymptotic behaviour of these eigen values as $n \rightarrow \infty$. Thus, for example, if $N(a, b ; n)$ is, for $n$ fixed, the number of $\lambda_{n, k}$ 's which satisfy $a \leqq \lambda_{n, k} \leqq b$, and if $\nu(y)$ is the Lebesgue measure of the set $\{\theta \mid t(\theta)<y\}$ then

$$
\lim _{n \rightarrow \infty} n^{-1} N(a, b ; n)=\nu(a)-\nu(b),
$$

provided $a$ and $b$ are points of continuity of $\nu$. This result was proved by Szegö, see [2; p. 64]. Detailed investigations have also been made of the behaviour of $\lambda_{n, k}$ as $n \rightarrow \infty$ while $k$ is fixed, under various additional assumptions on $t(\theta)$. Suppose that $t(\theta)$ is continuous for $\theta \in T$, has a unique absolute maximum at $\theta=0$, and that $t(\theta)$ is twice continuously differentiable in a neighborhood of $\theta=0$ with $t^{\prime \prime}(0)<0$.

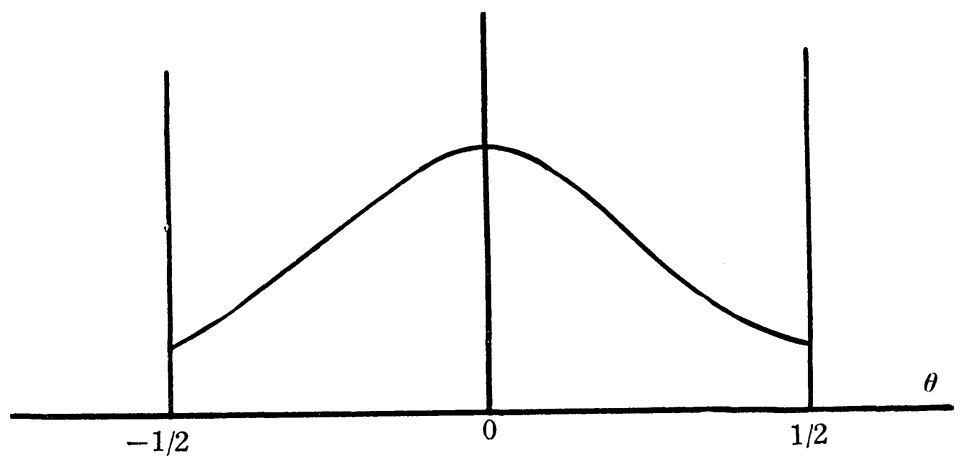

It was shown in 1953 by Kac, Murdock, and Szegö that under these assumptions

Received May 10, 1963. Research supported in part by the National Science Foundation under Grant No. G-24834. 


$$
\lambda_{n, k}=t(0)-\frac{t^{\prime \prime}(0)}{8} k^{2}(n+1)^{-2}+o\left(n^{-2}\right)
$$

as $n \rightarrow \infty$ for $k$ fixed, $k=1,2, \cdots$. In 1958 Widom, [14], proved that if $t(\theta)$ is even and four times continously differentiable near $\theta=0$ (in addition to the assumptions already made) then

$$
\lambda_{n, k}=t(0)-\frac{t^{\prime \prime}(0)}{8} k^{2}(n+1)^{-2}\left[1+\alpha(n+1)^{-1}\right]+o\left(n^{-3}\right)
$$

as $n \rightarrow \infty$, where

$$
\alpha=\int_{-1 / 2}^{1 / 2}\left[\csc ^{2} \pi \theta\right] \log \left[2 \pi^{2}\left(\frac{t(0)-t(\theta)}{t^{\prime \prime}(0)}\right) \operatorname{ctn}^{2} \pi \theta\right] d \theta .
$$

More recently Widom and Parter, see [9]-[11] and [15]-[17], have studied the behaviour of $\lambda_{n, k}$ under less restrictive assumptions on the nature of the maximum of $t(\theta)$. Suppose that $t(0)$ is again the unique maximum of $t(\theta)$, and that there exist constants $\sigma_{1}>0, \sigma_{2}>0$, and $\omega>0$ such that

$$
t(\theta) \sim \begin{cases}t(0)-\sigma_{1}|\theta|^{\omega} & \theta \longrightarrow 0+ \\ t(0)-\sigma_{2}|\theta|^{\omega} & \theta \longrightarrow 0-.\end{cases}
$$

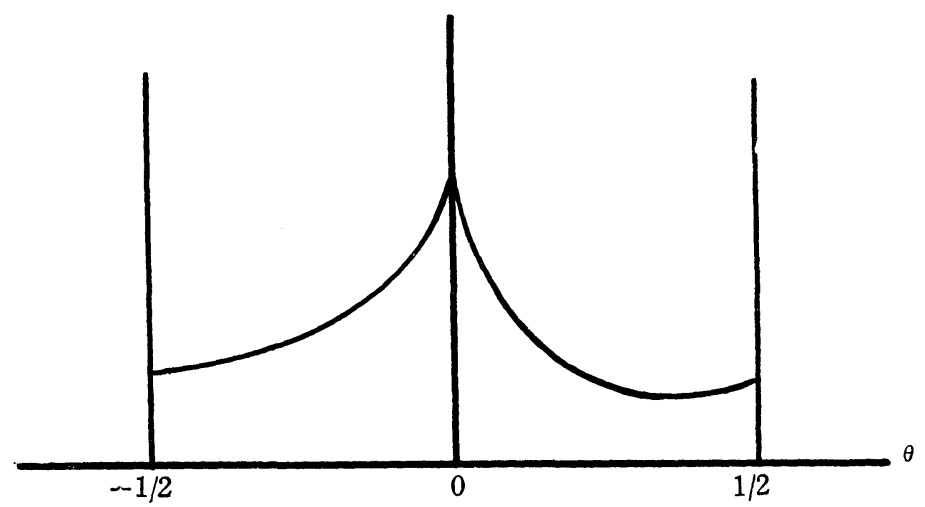

Then

$$
\lambda_{n, k}=t(0)-\mu_{k} n^{-\omega}+o\left(n^{-\omega}\right)
$$

where $0<\mu_{1} \leqq \mu_{2} \leqq \cdots, \lim _{k \rightarrow \infty} \mu_{k}=\infty$ are eigen values of a certain operator depending only on $\sigma_{1}, \sigma_{2}$, and $\omega$. The formula (4) evidently includes (2) as a very special case.

Let $\alpha, \beta>1$ be fixed and let

$$
2^{n} n ! P_{n}^{(\alpha \beta)}(x)=(-1)^{n}(1-x)^{-\alpha}(1+x)^{-\beta} D^{n}\left[(1-x)^{\alpha+n}(1+x)^{\beta+n}\right],
$$

where $D=d / d x$, be the Jacobi polynomial of order $n, n=0,1,2, \cdots$. The Jacobi polynomials are orthogonal on the interval $[-1,1]$ with 
respect to the weight function

$$
w_{\alpha, \beta}(x)=w(x)=(1-x)^{\alpha}(1+x)^{\beta} ;
$$

more precisely

$$
\int_{-1}^{1} P_{n}^{(\alpha, \beta)}(x) P_{m}^{(\alpha, \beta)}(x) w(x) d x=\delta(n, m) h_{n}
$$

where $\delta(n, m)$ is the Kronecker delta and where

$$
\begin{aligned}
(2 n+\alpha+\beta+1) & n ! \Gamma(n+\alpha+\beta+1) h_{n} \\
& =2^{\alpha+\beta+1} \Gamma(n+\alpha+1) \Gamma(n+\beta+1) .
\end{aligned}
$$

Let $t(x)$ be a real function in $L^{1}(w)$ and let

$$
c(j, k)=\left(h_{j} h_{k}\right)^{-1 / 2} \int_{-1}^{1} P_{j}^{(\alpha, \beta)}(x) P_{k}^{(\alpha, \beta)}(x) t(x) w(x) d x
$$

for $j, k=0,1, \cdots$. If

$$
C_{n}=[c(j, k)] \quad j, k=0, \cdots, n
$$

then $C_{n}$ is a generalized Toeplitz matrix of index $n$ associated with $t(x)$. Since $C_{n}$ is real and symmetric its eigen values $\left\{\lambda_{n, k}\right\}_{1}^{n+1}$ are real. In part the studies carried out for ordinary Toeplitz matrices have also been carried out for various classes of generalized Toeplitz matrices, and in particular for the generalized Toeplitz matrices constructed using Jacobi polynomials. For example, if we again define $N(a, b ; n)$ to be the numbers of $\lambda_{n, k}$ 's which satisfy $a<\lambda_{n, k} \leqq b$ and if $\nu(y)$ is $\pi^{-1}$ times the Lebesgue measure of the set $\{\theta \mid 0 \leqq \theta \leqq \pi, t(\cos \theta)<y\}$, then

$$
\lim _{n \rightarrow \infty} \frac{1}{n} N(a, b ; n)=\nu(a)-\nu(b)
$$

whenever $a$ and $b$ are points of continuity of $\nu(y)$. See [2; p. 114].

In [5] the author obtained formulas analgous to (2) and (3) but applying to generalized Toeplitz matrices constructed using the various classical orthogonal polynomials. Thus, for example, Let $t(x)$ be defined

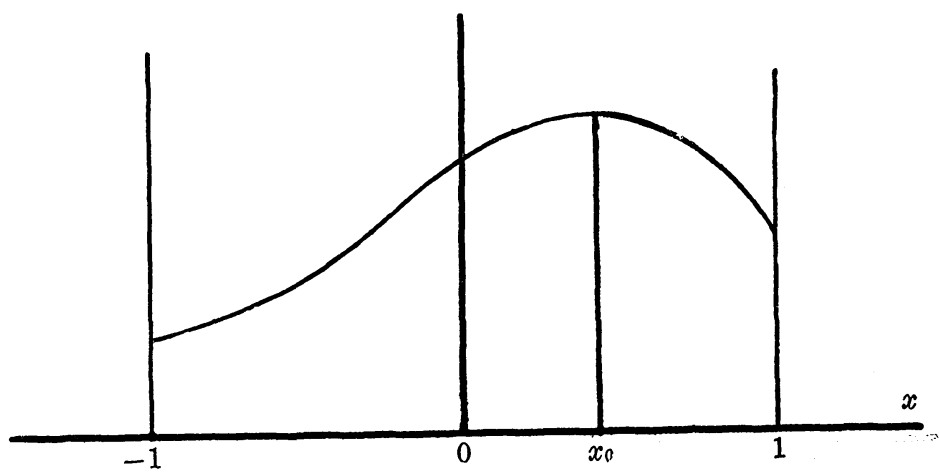


and continuous for $-1 \leqq x \leqq 1$, and have a unique absolute maximum at $x_{0},-1<x_{0}<1$. Let $t(x)$ be continuously differentiable in a neighborhood of $x_{0}$ and let $t^{\prime \prime}\left(x_{0}\right)=-\sigma^{2}<0$. If $C_{n}$ is the generalized Toeplitz matrix constructed from $t(x)$ using the Jacobi polynomials, then

$$
\lambda_{n, k}=t\left(x_{0}\right)-\left(1-x_{0}^{2}\right) \sigma^{2} k^{2} / 8 n^{2}+o\left(n^{-2}\right)
$$$$
\text { as } n \rightarrow \infty \text {. }
$$

Let $t(x)$ have a unique absolute maximum at $x=1$, let $t(x)$ be continuously differentiable in a neighborhood of $x=1$, and let $t^{\prime}(1)=\sigma>0$.

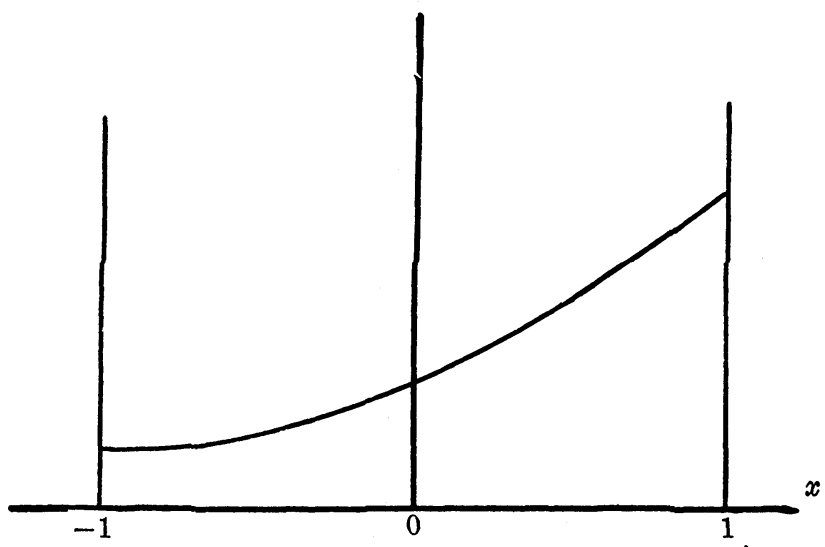

Then

$$
\lambda_{n, k}=t(1)-\frac{\sigma}{2}\left(\frac{z_{\alpha, k}}{n}\right)^{2}+o\left(n^{-2}\right)
$$

where $0<z_{\alpha, 1}<z_{\alpha, 2}<\cdots$, are the positive zeros of $J_{a}(z)$. See [5], where a more precise result analagous to (3) is also given.

In the present paper we will obtain formulas analogous to (4) for generalized Toeplitz matrices constructed using Jacobi polynomials. For example let $t(x)$ be continuous for $-1 \leqq x \leqq 1$, let the unique absolute maximum be at $x=1$, and let

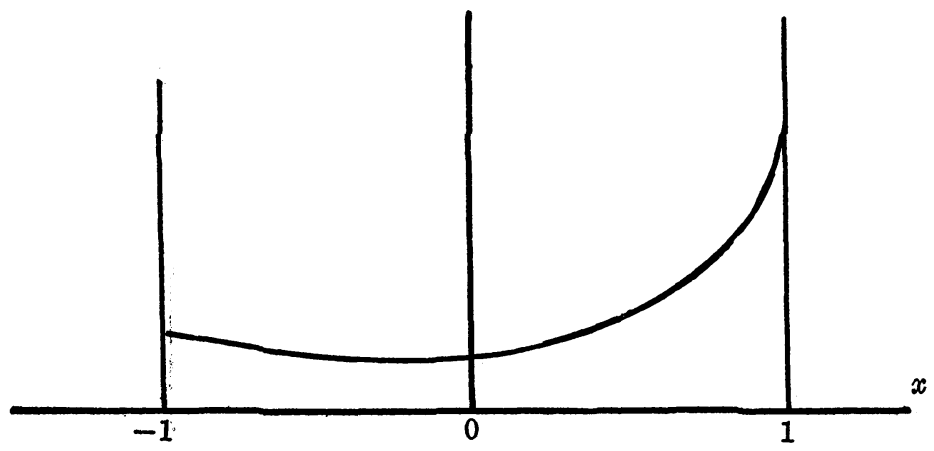




$$
t(x)-t(1)-\sigma(1-x)^{\omega}
$$

where $\sigma$ and $\omega$ are positive. We then have

$$
\lambda_{n, k}=t(1)-\mu_{k}(k / n)^{2 \omega}+o\left(n^{-2 \omega}\right)
$$

as $n \rightarrow \infty$ where $0<\mu_{1} \leqq \mu_{2} \leqq \cdots, \lim _{k \rightarrow \infty} \mu_{k}=\infty$, are the eigen values of an operator depending only upon $\sigma$ and $\omega$, and $\alpha$ but not otherwise upon $t(x)$ nor upon $\beta$. The case of a unique absolute maximum in the interior of $-1 \leqq x \leqq 1$ is also considered.

The program of demonstration of our results runs parallel to that employed in [17]. Sections 2-7 are devoted to developing an appropriate perturbation theory in Hilbert space. This theory is a rearticulation and partial generalization of the perturbation theory constructed by Widom. In sections $8-14$ and $15-19$ this theory is applied to the case where the maximum of $t(x)$ occurs at an end point of $-1 \leqq x \leqq 1$, and to the case where the maximum occurs at an interior point, respectively.

A large number of known properties of Jacobi polynomials, Jacobi functions of the second kind, Bessel functions, etc. are required in the course of this paper. Many of these results are collected in the Appendix.

2. A perturbation problem. Let $\boldsymbol{H}$ be a Hilbert space with elements $f, g, h$, etc. The inner product and norm in $\boldsymbol{H}$ are denoted by (|) and \| $\|$. Let $S$ and $S_{n}$ be unbounded self-adjoint operators in $\boldsymbol{H}$ with spectral resolutions.

$$
\begin{aligned}
S & =\int \lambda d \Phi(\lambda), \\
S_{n} & =\int \lambda d \Phi_{n}(\lambda) .
\end{aligned}
$$

If $S$ is the closure of the strong limit of the $S_{n}$ 's as $n \rightarrow \infty$ then Rellich's theorem asserts that in the strong operator topology

$$
\lim _{n \rightarrow \infty} \Phi_{n}(\lambda)=\Phi(\lambda)
$$

for every $\lambda,-\infty<\lambda<\infty$, not in the point spectrum of $S$. See $[13$, p. 56].

Let $F$ and $F_{n}$ be bounded not necessarily self-adjoint transformations of $\boldsymbol{H}$, such that $F$ is the strong limit of the $F_{n}$ 's as $n \rightarrow \infty$. In order to fix our attention suppose that the $S_{n}$ 's are bounded, but not necessarily $S$. Then for each $n S_{n, F}=F_{n}^{*} S_{n} F_{n}$ is a bounded selfadjoint transformation. Let its spectral resolution be 


$$
S_{n, F}=\int \lambda d \Psi_{n}(\lambda)
$$

Formally let $S_{F}=F^{*} S F$,

$$
S_{F}=\int \lambda d \Psi(\lambda)
$$

The problem we wish to study is that of passing from the convergence of the $S_{n}$ 's to $S$ and the $F_{n}$ 's to $F$ to the convergence of the $\Psi_{n}(\lambda)$ 's to $\Psi(\lambda)$. However there are several difficulties. First $F^{*} S F$ is not in general self-adjoint or even densely defined. Secondly the $S_{n, F}$ 's may not suitably converge to $S_{F}$. In $\S \S 3-6$ we will show essentially that if $0 \leqq S_{n} n=1,2, \cdots, 0 \leqq S$, (that is if the $S_{n}$ 's and $S$ are bounded from below) then these difficulties can be overcome.

Throughout we assume that the Hilbert space $\boldsymbol{H}$ is separable. while this is not at all necessary, it makes possible a simpler and more intuitive language.

3. Definition of $S_{F}$. We assume henceforth that:

i. $0 \leqq S$ is a self-adjoint operator on $\boldsymbol{H}$;

ii. $\boldsymbol{F}$ is a bounded operator on $\boldsymbol{H}$.

We define

$$
\boldsymbol{S}=\left\{f \mid F f \in \boldsymbol{D}\left(S^{1 / 2}\right)\right\} .
$$

Here $S^{1 / 2}$ is the unique positive square root of $S$ and $D\left(S^{1 / 2}\right)$ is its domain. We do not assume that $\boldsymbol{S}$ is dense in $\boldsymbol{H}$ although this is the most interesting special case. Let $\boldsymbol{M}$ be the closure of $\boldsymbol{S}$ in $\boldsymbol{H} . \boldsymbol{M}$ is a closed subspace of $\boldsymbol{H}$ and inherits the structure of a Hilbert space from $\boldsymbol{H}$. Our goal is to construct a self-adjoint transformation $S_{F}$ on the Hilbert space $\boldsymbol{M}$ with the properties:

$$
\begin{gathered}
D\left(S_{F}\right) \subset S ; \\
\left(S_{F} f \mid g\right)=\left(S^{1 / 2} F f \mid S^{1 / 2} F g\right)
\end{gathered}
$$

for all $f \in D\left(S_{F}\right)$ and for all $g \in S$. The construction of $S_{F}$ with these properties has long been known, see for example [13; p. 35], however it is included for the sake of completeness. We will need the following simple and well known fact which we record as a lemma.

LEMMA 3a. Let $A$ be a self-adjoint transformation on $\boldsymbol{H}$ and let $h_{n} \in \boldsymbol{D}(A) n=1,2, \cdots$. If

$$
h_{n} \rightarrow h
$$

as $n \rightarrow \infty$ 


$$
\left\|A h_{n}\right\|=O(1)
$$

as $n \rightarrow \infty$,

then $h \in \boldsymbol{D}(A)$ and $A h_{n} \rightarrow A h$.

Here " $\rightarrow$ " indicates strong convergence and " $\rightarrow$ " indicates weak convergence in $\boldsymbol{H}$. Lemma $3 \mathrm{a}$ is a special case of Lemma $4 \mathrm{a}$ which is proved in $\S 4$.

For $f, g \in \boldsymbol{S}$ let us define

$$
\begin{aligned}
\langle f \mid g\rangle & =\left(S^{1 / 2} F f \mid S^{1 / 2} F g\right)+(f \mid g), \\
\|\| f \| \mid & =\langle f \mid f\rangle^{1 / 2} .
\end{aligned}
$$

LEMMA 3b. With the definition of inner product and norm given by (3) $\boldsymbol{S}$ is a Hilbert space.

Proof. It is evident that $S$ is a pre-Hilbert space. We need only verify that $\boldsymbol{S}$ is complete. Suppose $f_{n} \in \boldsymbol{S} n=1,2, \cdots,\left\|\left|f_{n}-f_{m}\right|\right\| \rightarrow 0$ as $n, m \rightarrow \infty$. Since $\left\|f_{n}-f_{m}\right\| \leqq\left\|f_{n}-f_{m}\right\|$ there exists $f \in \boldsymbol{H}$ such that $\left\|f-f_{n}\right\| \rightarrow 0$ as $n \rightarrow \infty$. Since $\left\|S^{1 / 2} F\left(f_{n}-f_{m}\right)\right\| \leqq\left\|f_{n}-f_{m}\right\|$ there exists $g \in \boldsymbol{H}$ such that $\left\|S^{1 / 2} F f_{n}-g\right\| \rightarrow 0$ as $n \rightarrow \infty$. Applying Lemma $3 a$ with $h_{n}=F f_{n}$ and $A=S^{1 / 2}$ we see (since weak and strong limits coincide when both exist) that $F f \in D\left(S^{1 / 2}\right)$ and that $g=S^{1 / 2} F f$. Thus $f \in \boldsymbol{S}$ and

$$
\left\|f-f_{n}\right\|^{2}=\left\|S^{1 / 2} F\left(f-f_{n}\right)\right\|^{2}+\left\|f-f_{n}\right\|^{2} \rightarrow 0 \quad \text { as } n \rightarrow \infty .
$$

Lemma 3c. There exists a linear transformation $W$ of $\boldsymbol{M}$ into $\boldsymbol{S}$ such that $(f \mid g)=\langle f \mid W g\rangle$ for all $f \in \boldsymbol{S}, g \in \boldsymbol{M}$ and:

$$
\begin{array}{rll}
\text { i. } & \|W f\| \leqq\|W f\| \leqq\|f\| & \text { for all } f \in \boldsymbol{M} ; \\
\text { ii. } & (W f \mid g)=(f \mid W g) & \text { for all } f, g \in \boldsymbol{M} \text {; } \\
\text { iii. } & 0<(W f \mid f) & \text { for all } f \in \boldsymbol{M} .
\end{array}
$$

Proof. For $g \in \boldsymbol{M}$ fixed $(f \mid g)$ is a linear functional on $\boldsymbol{S}$ and since

$$
|(f \mid g)| \leqq\|f\|\|g\| \leqq\|f|\|\mid\| g \|
$$

$(f \mid g)$ is a bounded linear functional on $S$. Therefore there exists a unique element $g^{1} \in \boldsymbol{S}$ such that

$$
(f \mid g)=\left\langle f \mid g^{1}\right\rangle \quad \text { for all } f \in S .
$$

Clearly the mapping $g \rightarrow g^{1}$ defines a linear transformation of $\boldsymbol{M}$ into $\boldsymbol{S}, g^{1}=W g$. It is evident that $\|W g\| \mid\|g\|$ so that i. holds. Suppose that $f, g \in S$. Then

$$
(W f \mid g)=\langle W f \mid W g\rangle=(f \mid W g)
$$


so that ii. is valid if $f, g \in S$. By continuity it is also valid for $f, g \in M$. Thus $W$ is a self-adjoint transformation on $\boldsymbol{M}$. Since

$$
(W f \mid f)=\langle W f \mid W f\rangle>0 \quad f \in \boldsymbol{S}
$$

and since $\boldsymbol{S}$ is dense in $\boldsymbol{M}$ we have $0 \leqq W$. To show that $0<W$ we need only verify that $W f=0$ is impossible unless $f=0$. If $W f=0$, then

$$
(g \mid f)=\langle g \mid W f\rangle=0 \quad \text { for all } g \in S,
$$

but since $S$ is dense in $\boldsymbol{M}$ this implies that $f=0$.

THEOREM 3d. There exists a self-adjoint operator $S_{F}$ on $M$ satisfying conditions (1) and (2).

Proof. We define

$$
S_{F^{\prime}}=W^{-1}-I \text {. }
$$

It is evident from this definition that $S_{F}$ is a self-adjoint operator, and that

$$
D\left(S_{F}\right)=D\left(W^{-1}\right)=R(W) \subset S,
$$

where $\boldsymbol{R}(W)$ is the range of $W$. If $f \in \boldsymbol{D}\left(S_{F}\right)$ and $g \in \boldsymbol{S}$ then

$$
\begin{aligned}
\left(S_{F} f \mid g\right) & =\left(W^{-1} f \mid g\right)-(f \mid g)=\langle f \mid g\rangle-(f \mid g), \\
& =\left(S^{1 / 2} F f \mid S^{1 / 2} F g\right),
\end{aligned}
$$

and our proof is complete.

4. The resolvant relation. Let $A$ be a closed linear operator on $\boldsymbol{M}$. It is not assumed that $\boldsymbol{D}(A)$ is dense in $\boldsymbol{M}$. A subset $\boldsymbol{C} \subset \boldsymbol{D}(A)$ is said to be a core for $A$ if the set $\{(f, g) \mid g=A f, f \in \boldsymbol{C}\}$ in $\boldsymbol{H} \times \boldsymbol{H}$ is dense in the set $\{(f, g) \mid g=A f, f \in D(A)\}$. Let $A_{n}$ and $A$ be closed linear operators in $\boldsymbol{H}$ and let $\boldsymbol{C}=\left\{f \mid A_{n} f \rightarrow A f\right.$ as $\left.n \rightarrow \infty\right\}$. If $\boldsymbol{C}=$ $\boldsymbol{D}(A)$ we say that $A$ is the strong limit of the $A_{n}$ 's; if $C$ is a core for $A$ we say that $A$ is the closure of the strong limit of the $A_{n}$ 's.

LEMMA 4a. Let $A_{n}$ and $A$ be self-adjoint operators on $\boldsymbol{H}$ and let $A$ be the closure of the strong limit of the $A_{n}$ 's. Then if

$$
f_{n} \rightarrow f, \quad\left\|A_{n} f_{n}\right\|=O(1),
$$

we have

$$
f \in D(A) \text { and } A_{n} f_{n} A \rightarrow f .
$$

Proof. We denote by $\mathfrak{p}$ the positive integers $\{1,2,3, \cdots\}$. A 
subsequence $\mathfrak{p}_{1}$ of $\mathfrak{p}$ is then a subset $\left\{n_{1}, n_{2}, n_{3} \cdots\right\}$ of $\mathfrak{p}$ with $1 \leqq$ $n_{1}<n_{2}<\cdots$. By " $a_{n} \rightarrow a$ as $n \rightarrow \infty$ in $\mathfrak{p}_{1}$ " we mean that $\lim _{k \rightarrow \infty} a_{n_{k}}=$ a. This notation enables us to dispense with awkward subscripts.

Let $C=\left\{f \mid A_{n} f \rightarrow A f\right.$ as $\left.n \rightarrow \infty\right\}$. By assumption $C$ is a core for $A$. Since $\left\|A_{n} f_{n}\right\|=O(1)$ given any subsequence $\mathfrak{p}_{1}$ of $\mathfrak{p}$ there exists a subsequence $\mathfrak{p}_{2}$ of $\mathfrak{p}_{1}$ such that $A_{n} f_{n} \rightarrow g$ as $n \rightarrow \infty$ in $\mathfrak{p}_{2}$ for some $g \in \boldsymbol{H}$. This is because bounded sets in $\boldsymbol{H}$ are weakly conditionally compact. In particular if $h \in C$ then

$$
\left(A_{n} f_{n} \mid h\right) \rightarrow(g \mid h) \quad \text { as } n \rightarrow \infty \text { in } \mathfrak{p}_{2} \text {. }
$$

On the other hand

$$
\left(A_{n} f_{n} \mid h\right)=\left(f_{n} \mid A_{n} h\right)
$$

for all (large) $n$ and thus

$$
\left(A_{n} f_{n} \mid h\right) \rightarrow(f \mid A h)
$$

as $n \rightarrow \infty$ in $\mathfrak{p}_{2}$,

so that

$$
(g \mid h)=(f \mid A h) .
$$

Given $k \in \boldsymbol{D}(A)$ and $\delta>0$ there exists $h \in \boldsymbol{C}$ such that $\|k-h\|<\delta$, $\|A k-A h\|<\delta$. This implies that

$$
(g \mid k)=(f \mid A k) \quad \text { for all } k \in \boldsymbol{D}(A) .
$$

Consequently $f \in \boldsymbol{D}\left(A^{*}\right)$ and $A^{*} f=g$; but $A^{*}=A$. Since every subsequence $\mathfrak{p}_{1}$ contains a subsequence $\mathfrak{p}_{2}$ such that $A_{n} f_{n} \rightarrow A f$ as $n \rightarrow \infty$ in $\mathfrak{p}_{2}$ it follows that $A_{n} f_{n} \rightarrow A f$ as $n \rightarrow \infty$ in $\mathfrak{p}$.

In what follows we assume that:

iii. $O \leqq S_{n}$ is a self-adjoint transformation on $\boldsymbol{H} n=1,2, \cdots ; \boldsymbol{F}_{n}$ is a bounded transformation on $\boldsymbol{H} n=1,2, \cdots ; \boldsymbol{R}\left(F_{n}\right) \subset \boldsymbol{D}\left(S_{n}\right) n=$ $1,2, \cdots$. Assumption iii. implies that $S_{n, F}=F_{n}^{*} S_{n} F_{n}$ is a bounded operator on $\boldsymbol{H}$ for $n=1,2, \cdots$. We further assume that:

iv. $F$ is a bounded operator on $\boldsymbol{H}$ and $F$ is the strong limit of $F_{n}$ as $n \rightarrow \infty$;

v. $S^{1 / 2}$ is the closure of the strong limit of $S_{n}^{1 / 2}$ as $n \rightarrow \infty$;

vi. $S^{1 / 2} F$ is the closure of the strong limit of $S_{n}^{1 / 2} F_{n}$ as $n \rightarrow \infty$. We set

$$
S^{\prime}=\left\{f \mid S_{n}^{1 / 2} F_{n} f \rightarrow S^{1 / 2} F f \text { as } n \rightarrow \infty\right\} \text {. }
$$

It is evident that $\boldsymbol{S}^{\prime} \subset \boldsymbol{S}$.

THEOREM 4b. Under assumptions $\mathrm{i}-\mathrm{vi}$ if there exists $\delta>0$ such that dist $\left\{z, \sigma\left(S_{F}\right)\right\} \geqq \delta$, dist $\left\{z, \sigma\left(S_{n, F}\right)\right\} \geqq \delta, n=1,2, \cdots$, then for all $f \in \boldsymbol{M}$ 


$$
\left\{S_{n, F}-z I\right\}^{-1} f \rightarrow\left\{S_{F}-z I\right\}^{-1} f \quad \text { as } n \rightarrow \infty
$$

Proof. Take $f \in \boldsymbol{M}$. We will show that if $\mathfrak{p}_{1}$ is an arbitrary subsequence of $\mathfrak{p}$ then $\mathfrak{p}_{1}$ contains a subsequence $\mathfrak{p}_{2}$ such that

$$
\left\{S_{n, F}-z I\right\}^{-1} f \rightarrow\left\{S_{F}-z I\right\}^{-1} f \quad \text { as } n \rightarrow \infty \text { in } \mathfrak{p}_{2} \text {. }
$$

This will prove our result. Because $\operatorname{dist}\left\{z, \sigma\left(S_{n, F}\right)\right\} \geqq \delta$ it follows that $\left\|\left\{S_{n, F}-z I\right\}^{-1} f\right\|=0(1)$ as $n \rightarrow \infty$. Therefore we can find a subsequence $\mathfrak{p}_{2}$ of $\mathfrak{p}_{1}$ such that if $g_{n}=\left\{S_{n, F}-z I\right\}^{-1} f$ then $g_{n} \rightarrow g$ as $n \rightarrow \infty$ in $\mathfrak{p}_{2}$ for some $g \in \boldsymbol{H}$. We must show that $g=\left\{S_{F}-z I\right\}^{-1} f$. Since $F$ is. the strong limit of $F_{n}$ we have

$$
F_{n} g_{n} \rightarrow F g \quad \text { as } n \rightarrow \infty \text { in } \mathfrak{p}_{2},
$$

and since $S_{n, F} g_{n}=f+z g_{n}$ we have

$$
\begin{aligned}
\left(S_{n}^{1 / 2} F_{n} g_{n} \mid S_{n}^{1 / 2} F_{n} g_{n}\right) & =\left(S_{n, F} g_{n} \mid g_{n}\right) \\
& =\left(f+z g_{n} \mid g_{n}\right)=O(1) .
\end{aligned}
$$

Therefore by Lemma $4 \mathrm{a} F g \in D\left(S^{1 / 2}\right)$ and $S_{n}^{1 / 2} F_{n} g_{n} \rightarrow S^{1 / 2} F g$ as $n \rightarrow \infty$ in $\mathfrak{p}_{2}$. In particular $g \in S$. Take $h \in \boldsymbol{S}^{\prime}$; then by the above

$$
\begin{aligned}
\lim _{\mathfrak{p}_{2}}\left(S_{n, F} g_{n} \mid h\right) & =\lim _{\mathfrak{p}_{2}}\left(S_{n}^{1 / 2} F_{n} g_{n} \mid S_{n}^{1 / 2} F_{n} h\right) \\
& =\left(S^{1 / 2} F g \mid S^{1 / 2} F h\right) .
\end{aligned}
$$

On the other hand

$$
\lim _{\mathfrak{p}_{2}}\left(S_{n, F} g_{n} \mid h\right)=\lim _{\mathfrak{p}_{2}}\left(f+z g_{n} \mid h\right)=(f+z g \mid h) .
$$

Thus

$$
\left(S^{1 / 2} F g \mid S^{1 / 2} F h\right)=(f+z g \mid h)
$$

for all $h \in S^{\prime}$. Since $S^{\prime}$ is by assumption a core for $S^{1 / 2} F$ (1) holds. for all $h \in \boldsymbol{S}$, and thus for all $h \in \boldsymbol{D}\left(S_{F}\right)$. For such an $h$ we have

$$
\left(S^{1 / 2} F g \mid S^{1 / 2} F h\right)=\left(g \mid S_{F} h\right)
$$

by Theorem 3d. Consequently we have shown that

$$
\left(g \mid S_{F} h\right)=(f+z g \mid h)
$$

or equivalently

$$
\left(g \mid\left\{S_{F}-z^{*} I\right\} h\right)=(f \mid h)
$$

for all $h \in \boldsymbol{D}\left(S_{F}\right)$. This implies that

$$
\left\{S_{F}-z^{*} I\right\}^{*} g=f
$$


and hence that

$$
g=\left\{S_{F}-z I\right\}^{-1} f
$$

5. Spectral resolutions. Let

$$
S_{n, F}=\int_{0-}^{\infty} \lambda d \Psi_{n}(\lambda)
$$

be the spectral resolution of $S_{n, F}$ on $\boldsymbol{H}$ and

$$
S_{F}=\int_{0-}^{\infty} \lambda d \Psi(\lambda)
$$

be the spectral resolution of $S_{F}$ on $\boldsymbol{M}$. We assume throughout that $\Psi_{n}(\lambda)=\Psi_{n}(\lambda+), 0 \leqq \lambda<\infty, n=1,2, \cdots$, that $\Psi_{n}(0-)=0$, and similarly for $\Psi(\lambda)$.

THEOREM 5a. If $\lambda \geqq 0$ is not in the point spectrum of $S_{F}$ and if $f \in \boldsymbol{M}$ then

$$
\Psi_{n}(\lambda) f \rightarrow \Psi(\lambda) f \quad \text { as } n \rightarrow \infty \text {. }
$$

Proof. Fix $f \in \boldsymbol{M}$, and let $h=\Psi(\lambda) f, h_{n}=\Psi_{n}(\lambda) f$. It is enough to show that if $\mathfrak{p}_{1}$ is any sequence such that $h_{n} \rightarrow h^{\prime}$ as $n \rightarrow \infty$ in $\mathfrak{p}_{1}$, then $h^{\prime}=h$. In order to identify $h^{\prime}$ we proceed as follows. We assert that if $g \in \boldsymbol{H}$ then

$$
\begin{aligned}
\lim _{\mathfrak{p}}(2 \pi i)^{-1} \int_{\sigma}\left(\left\{S_{n, F}-z I\right\}^{-1} f \mid g\right)(\lambda-z) d z \\
=(2 \pi i)^{-1} \int_{\sigma}\left(\left\{S_{F^{F}}-z I\right\}^{-1} f \mid g\right)(\lambda-z) d z,
\end{aligned}
$$

where $C$ is the curve pictured below.

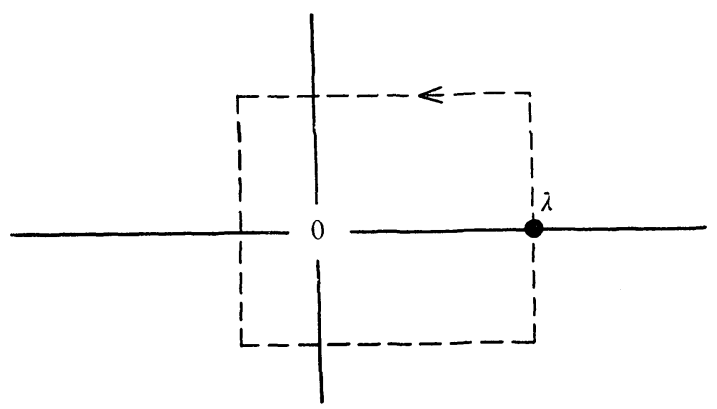

Indeed by Theorem $4 \mathrm{~b}$

$$
\lim _{\mathfrak{p}}\left(\left\{S_{n, F}-z I\right\}^{-1} f \mid g\right)=\left(\left\{S_{F}-z I\right\}^{-1} f \mid g\right)
$$


for all $z$ on $C$ except $z=\lambda$. Moreover starting from the inequality $\left\|(A-z I)^{-1}\right\| \leqq 1 /$ dist $\{z, \sigma(A)\}$ one can easily show that for $z$ on $C$ and some constant $k(C)$

$$
\left|\left(\left\{S_{n, F}-z I\right\}^{-1} f \mid g\right)\right| \leqq k(C)|\lambda-z|^{-1}|| f|||g| \mid \text {. }
$$

Applying the Lebesgue limit theorem we obtain (1).

A simple computation gives

$$
(2 \pi i)^{-1} \int_{\sigma} \frac{\lambda-z}{\mu-z} d z=\left\{\begin{array}{cll}
\mu-\lambda & \text { if } & 0 \leqq \mu \leqq \lambda \\
0 & \text { if } \quad \mu>\lambda .
\end{array}\right.
$$

We have

$$
\begin{aligned}
(2 \pi i)^{-1} \int_{\sigma}\left(\left\{S_{F}-z I\right\}^{-1} f \mid g\right)(\lambda-z) d z & \\
& =(2 \pi i)^{-1} \int_{0}(\lambda-z) d z \int_{0-}^{\infty}(\mu-z)^{-1} d_{\mu}(\Psi(\mu) f \mid g) .
\end{aligned}
$$

This iterated integral is absolutely convergent and therefore using Fubini's theorem and (2) we obtain

$$
\begin{aligned}
(2 \pi i)^{-1} \int_{\sigma} & \left(\left\{S_{F}-z I\right\}^{-1} f \mid g\right)(\lambda-z) d z=\int_{0-}^{\lambda}(\mu-\lambda) d_{\mu}(\Psi(\mu) f \mid g) \\
& =\left(\left\{S_{F}-\lambda I\right\} \Psi(\lambda) f \mid g\right) \\
& =\left(\left\{S_{F}-\lambda I\right\} h \mid g\right) .
\end{aligned}
$$

Similarly

$$
\begin{gathered}
(2 \pi i)^{-1} \int_{0}\left(\left\{S_{n, F^{*}}-z I\right\}^{-1} f \mid g\right)(\lambda-z) d z=\left(\left\{S_{n, F^{*}}-\lambda I\right\} \Psi_{n}(\lambda) f \mid g\right) \\
\quad=\left(\left\{S_{n, F^{*}}-\lambda I\right\} h_{n} \mid g\right) .
\end{gathered}
$$

Using (1), (3), and (4) we see that

$$
\left(\left\{S_{n, F}-\lambda I\right\} h_{n} \mid g\right) \rightarrow\left(\left\{S_{F}-\lambda I\right\} h \mid g\right) \quad \text { as } n \rightarrow \infty \text { in } \mathfrak{p} .
$$

Since $h_{n}=\Psi_{n}(\lambda) f$ it follows that

$$
\begin{aligned}
\left(S_{n}^{1 / 2} F_{n} h_{n} \mid S_{n}^{1 / 2} F_{n} h_{n}\right) & =\left(S_{n, F} h_{n} \mid h_{n}\right) \\
& =\left(S_{n, F} \Psi_{n}(\lambda) f \mid \Psi_{n}(\lambda) f\right) \\
& =\int_{0-}^{\lambda} \mu d_{\mu}\left\|\Psi_{n}(\mu) f\right\|^{2} \\
& \leqq \lambda\|f\|^{2} .
\end{aligned}
$$

We also have, since $F$ is the strong limit of $F_{n}$, that

$$
F_{n} h_{n} \rightarrow F h^{\prime} \quad \text { as } n \rightarrow \infty \text { in } \mathfrak{p}_{1} .
$$

Applying Lemma $4 \mathrm{a}$ we find that $h^{\prime} \in S$ and that $S_{n}^{1 / 2} F_{n} h_{n} \rightarrow S^{1 / 2} F h^{\prime}$ 
as $n \rightarrow \infty$ in $\mathfrak{p}_{1}$. Suppose that $g \in S$; then

$$
\left(S_{n, F} h_{n} \mid g\right)=\left(S_{n}^{1 / 2} F_{n} h_{n} \mid S_{n}^{1 / 2} F_{n} g\right)
$$

and thus

$$
\left(S_{n, F} h_{n} \mid g\right) \rightarrow\left(S^{1 / 2} F h^{\prime} \mid S^{1 / 2} F g\right)
$$

also

$$
\left(S_{F} h \mid g\right)=\left(S^{1 / 2} F h \mid S^{1 / 2} F g\right) .
$$

Inserting (6) and (7) in (5) we find that

$$
\left(S^{1 / 2} F h^{\prime} \mid S^{1 / 2} F g\right)-\lambda\left(h^{\prime} \mid g\right)=\left(S^{1 / 2} F h \mid S^{1 / 2} F g\right)-\lambda(h \mid g)
$$

for all $g \in S^{\prime}$. Using assumption vi. we see that (8) holds for all $g \in \boldsymbol{S}$ and therefore in particular for all $g \in \boldsymbol{D}\left(S_{F}\right)$. Appealing to Theorem $3 \mathrm{~d}$ we obtain

$$
\left(h^{\prime}-h \mid S_{F} g\right)=\lambda\left(h^{\prime}-h \mid g\right)
$$

for all $g \in \boldsymbol{D}\left(S_{F}\right)$. Since $h^{\prime}-h \in \boldsymbol{S} \subset \boldsymbol{M}$ this implies that

$$
S_{F}\left(h^{\prime}-h\right)=\lambda\left(h^{\prime}-h\right) \text {. }
$$

However by assumption $\lambda$ is not in the point spectrum of $S_{F^{\prime}}$ so that $h^{\prime}-h=0$ and our proof is complete.

6. The perturbation theorem. In this section and also in $\S 7$ we make the following convention. Suppose that $\boldsymbol{P}$ is a subspace of $\boldsymbol{H}$. If $E$ is a projection of $\boldsymbol{P}$ onto a subspace $\boldsymbol{Q}$ of $\boldsymbol{P}$ then $E$ may also be regarded as projection of $\boldsymbol{H}$, namely the projection of $\boldsymbol{H}$ onto $\boldsymbol{Q}$.

THEOREM 6a. Under assumptions i-vi we have for every $f \in \boldsymbol{H}$

$$
\Psi_{n}(\lambda) f \rightarrow \Psi(\lambda) f
$$$$
\text { as } n \rightarrow \infty \text {, }
$$

for every $\lambda$ not in the point spectrum of $S_{F}$.

Proof. It follows from Theorem 5a that

$$
\Psi_{n}(\lambda) f \rightarrow \Psi(\lambda) f
$$

as $n \rightarrow \infty$,

for all $f \in \boldsymbol{M}$. Suppose next that $g \perp \boldsymbol{M}$. Since $\left\|\Psi_{n}(\lambda) g\right\|=0(1)$, given any subsequence $\mathfrak{p}_{1}$ there is a subsequence $\mathfrak{p}_{2}$ of $\mathfrak{p}_{1}$ such that

$$
\Psi_{n}(\lambda) g \rightarrow h \quad \text { as } n \rightarrow \infty \text { in } \mathfrak{p}_{2}
$$

for some $h \in \boldsymbol{H}$. If $f \in \boldsymbol{M}$ then

$$
\lim _{\mathfrak{p}_{2}}\left(\Psi_{n}(\lambda) g \mid f\right)=(h \mid f) .
$$


Since $\left(\Psi_{n}(\lambda) f \mid g\right)=\left(f \mid \Psi_{n}(\lambda) g\right)$ we have using (1)

$$
\lim _{p_{2}}\left(\Psi_{n}(\lambda) g \mid f\right)=(g \mid \Psi(\lambda) f)=0 \text {. }
$$

Thus $(h \mid f)=0$; i.e. $h \perp \boldsymbol{M}$. Now

$$
\begin{aligned}
\left\|S_{n}^{1 / 2} F_{n} \Psi_{n}(\lambda) g\right\|^{2} & =\left(S_{n, F} \Psi_{n}(\lambda) g \mid g\right), \\
& =\int_{0-}^{\lambda} \mu d_{\mu}\left\|\Psi_{n}(\mu) g\right\|^{2}, \\
& \leqq \lambda\|g\|^{2} .
\end{aligned}
$$

Therefore by Lemma 4a $F h \in \boldsymbol{D}\left(S^{1 / 2}\right)$; that is, $h \in \boldsymbol{S} \subset \boldsymbol{M}$. But $h \perp \boldsymbol{M}$ so that $h=0$. We have thus shown that

$$
\Psi_{n}(\lambda) g \rightarrow 0
$$

as $n \rightarrow \infty$ if $g \perp M$

The relations (1) and (2) together prove that

$$
\Psi_{n}(\lambda) f \rightarrow \Psi(\lambda) f
$$

as $n \rightarrow \infty$,

for all $f \in \boldsymbol{H}$. Since weak convergence of projections implies strong convergence our proof is complete.

7. Convergence in dimension. In this section we will show how starting from the conclusion of Theorem 6 a and one further assumption it is possible to prove that the dimensions of the spectral projections converge. Suppose that $0 \leqq R_{n} \quad n=1,2, \cdots$ are bounded self-adjoint operators defined on subspaces $N_{n}$ of a Hilbert space $\boldsymbol{H}$. Let $0 \leqq R$ be a self-adjoint operator on a subspace $\boldsymbol{N}$ of $\boldsymbol{H}$. Let

$$
\begin{aligned}
R_{n} & =\int_{0-}^{\infty} \lambda d E_{n}(\lambda), \\
R & =\int_{0-}^{\infty} \lambda d E(\lambda),
\end{aligned}
$$

be the spectral resolutions of $R_{n}$ on $N_{n}$ and of $R$ on $N$. We list two conditions.

a. $E_{n}(\lambda) \rightarrow E(\lambda)$ as $n \rightarrow \infty$ for all $\lambda>0, \lambda \notin \sigma_{p}(R)$, the point spectrum of $R$. Here " $\rightarrow$ " is in $\boldsymbol{H}$.

b. there is a number $m>0$ such that if $f_{n} \in N_{n},\left\|f_{n}\right\|=1$, and $\left(R_{n} f_{n} \mid f_{n}\right) \leqq m_{1}<m$ for $n \in \mathfrak{p}_{1}$, then $\mathfrak{p}_{1}$ contains a subsequence $\mathfrak{p}_{2}$ such that $f_{n} \rightarrow f \neq 0$ as $n \rightarrow \infty$ in $\mathfrak{p}_{2}$. Here " $\rightarrow$ " is in $\boldsymbol{H}$.

THEOREM 7a. Under assumptions a. and b. we have

$$
\operatorname{dim} E(\lambda)<\infty \quad 0 \leqq \lambda<m,
$$




$$
\lim _{n \rightarrow \infty} \operatorname{dim} E_{n}(\lambda)=\operatorname{dim} E(\lambda)
$$

for $0 \leqq \lambda<m, \lambda \notin \sigma_{p}(R)$.

Proof. We first note that assumption a. alone implies that if $0 \leqq \lambda<\infty, \lambda \notin \sigma_{p}(R)$, then

$$
\lim _{n \rightarrow \infty} \operatorname{dim} E_{n}(\lambda) \geqq \operatorname{dim} E(\lambda) \text {. }
$$

In (3) we admit " $\infty \geqq \infty$ ". Suppose $\operatorname{dim} E(\lambda) \geqq k$. Then there exist orthonormal vectors $g_{1}, g_{2}, \cdots, g_{k}$ in $E(\lambda) H$. By assumption a. we have

$$
\lim _{n \rightarrow \infty} E_{n}(\lambda) g_{j}=E(\lambda) g_{j}=g_{j} \quad j=1, \cdots, k,
$$

from which it follows that for all sufficiently large $n\left\{E_{n}(\lambda) g_{j}\right\}_{1}^{k}$, which belong to $E_{n}(\lambda) \boldsymbol{N}_{n}$, are linearly independant.

From this point on we use assumptions a. and b. We suppose that $\lambda \notin \sigma_{p}(R)$ and that $0 \leqq \lambda<m$. If $\operatorname{dim} E(\lambda)=\infty$ then we can find an infinite orthonormal set of vectors $\left\{g_{j}\right\}_{1}^{\infty}$ in $E(\lambda) H$. Using a. we see that there exists a subsequence $\mathfrak{p}_{1}=\left\{0<n_{1}<n_{2}<\cdots\right\}$ such that

$$
\left\|E_{n_{k}}(\lambda) g_{k}-g_{k}\right\| \rightarrow 0 \quad \text { as } k \rightarrow \infty .
$$

If we set $f_{n_{k}}=E_{n_{k}}(\lambda) g_{k} /\left\|E_{n_{k}}(\lambda) g_{k}\right\|$ then $f_{n}$ is defined for $n \in p_{1}$. We have $f_{n} \in N_{n},\left\|f_{n}\right\|=1$, and $\left(R_{n} f_{n} \mid f_{n}\right) \leqq \lambda$ for $n \in \mathfrak{p}_{1}$. Therefore by b. there is a subsequence $\mathfrak{p}_{2}$ of $\mathfrak{p}_{1}$, such that $f_{n} \rightarrow f$ as $n \rightarrow \infty$ in $\mathfrak{p}_{2}$, and $f \neq 0$. But then $g_{n} \rightarrow f \neq 0$ as $n \rightarrow \infty$ in $\mathfrak{p}_{2}$. However it is obvious that $g_{n} \rightarrow 0$ as $n \rightarrow \infty$. Thus $\operatorname{dim} E(\lambda)=\infty$ leads to a contradiction and (1) is true.

We assert that (2) is true. Set $k=\operatorname{dim} E(\lambda)$. If (2) is not true then in view of (3) there is a subsequence $\mathfrak{p}_{1}$ such that $\operatorname{dim} E_{n}(\lambda)>k$ for $n \in \mathfrak{p}_{1}$. Let $g_{1}, \cdots, g_{k}$ be an orthonormal basis for $E(\lambda) H$. For each $n \in \mathfrak{p}_{1}$ we can choose $f_{n} \in E_{n}(\lambda) N_{n}$ such that $\left\|f_{n}\right\|=1, f_{n} \perp g_{1}, \cdots$, $g_{k}$. We have $\left(R_{n} f_{n} \mid f_{n}\right) \leqq \lambda$ and therefore by b. there is a subsequence $\mathfrak{p}_{2}$ of $\mathfrak{p}_{1}$ such that $f_{n} \rightarrow f \neq 0$ as $n \rightarrow \infty$ in $\mathfrak{p}_{2}$. Now $f_{n}=E_{n}(\lambda) f_{n}$ and by a. $E_{n}(\lambda) f_{n} \rightarrow E(\lambda) f$ as $n \rightarrow \infty$ in $\mathfrak{p}_{2}$. Therefore $f=E(\lambda) f$ and $f \in E(\lambda) H$. Since $f \perp g_{1}, \cdots, g_{k} f$ must be 0 . This is a contradiction and our assertion follows.

8. Maximum at the end point. As we announced in the introduction $\S \S 8-14$ are devoted to the case in which $t(x)$ has a unique absolute maximum at $x=1$. We assume that $t(x)$ is continuous for $-1 \leqq x \leqq 1$ and that 


$$
t(x)<t(1)
$$$$
-1 \leqq x<1 .
$$

We further assume that

$$
t(1)-t(x)-(1-x)^{\omega} L(1-x) \quad \text { as } x \rightarrow 1-.
$$

Here $\omega>0$, and $L(y)$, defined for $0<y \leqq 2$, is positive, continuous, and slowly oscillating as $y \rightarrow 0+$. We recall that $L(y)$ "slowly oscillating" means that for every $\varepsilon>0, L(y) y^{\varepsilon}$ is increasing and $L(y) y^{-\varepsilon}$ is decreasing for $0<y<a(\varepsilon)$ if $a(\varepsilon)$ is sufficiently small.

In what follows it will be necessary for us to work with four Hilbert spaces. The first Hilbert space is $\boldsymbol{L}$, the elements of which are complex functions $f(k)$ defined for $k=0,1, \cdots$, with inner product

$$
(f \mid g)_{L}=\sum_{k=0}^{\infty} f(k) g(k)^{*} .
$$

The second Hilbert space is $\boldsymbol{L}^{\wedge}$ the elements of which are complex measurable functions on $-1 \leqq x \leqq 1$ with inner product defined by

$$
(f \mid g)_{L^{\wedge}}=\int_{-1}^{1} f(x) g(x)^{*} w_{\alpha, \beta}(x) d x .
$$

Here $w_{\alpha, \beta}(x)=w(x)=(1-x)^{\alpha}(1+x)^{\beta}, \alpha>-1, \beta>-1$. The mapping $\phi$ from $\boldsymbol{L}$ to $\boldsymbol{L}^{\wedge}$ defined by

$$
\phi f \cdot(x)=\sum_{k=0}^{\infty} f(k) h_{k}^{-1 / 2} P_{k}^{(\alpha, \beta)}(x)
$$

(the partial sums of this series converge in the metric of $\boldsymbol{L}^{\wedge}$ ) and its and its inverse $\phi^{-1}$ from $\boldsymbol{L}^{\wedge}$ to $\boldsymbol{L}$ defined by

$$
\phi^{-1} f \cdot(k)=\int_{-1}^{1} f(x) h_{k}^{-1 / 2} P_{k}^{(\alpha, \beta)}(x) w_{\alpha, \beta}(x) d x,
$$

are unitary mappings. Both $\boldsymbol{H}$ and $\boldsymbol{H}^{\wedge}$ have as elements complex measurable functions on $[0, \infty)$ with inner products

$$
\begin{aligned}
(f \mid g)_{\boldsymbol{H}^{\wedge}} & =\int_{0}^{\infty} f(u) g(u)^{*} u d u, \\
(f \mid g)_{\boldsymbol{H}} & =\int_{0}^{\infty} f(z) g(z)^{*} z d z .
\end{aligned}
$$

The mapping $\psi$ from $\boldsymbol{H}$ to $\boldsymbol{H}^{\wedge}$ defined by

$$
\psi f \cdot(u)=\int_{0}^{\infty} f(z) J_{\alpha}(z u) z d z
$$

(the partial integrals converge in the metric of $\boldsymbol{H}^{\wedge}$ ) and its inverse

$$
\psi^{-1} f \cdot(z)=\int_{0}^{\infty} f(u) J_{\alpha}(u z) u d u
$$


are also unitary mappings. See in this connection $[1 ; p .73]$ and the references given there.

Let us set

$$
\theta_{n}^{(\alpha, \beta)}(u)=\left(1-\cos \frac{u}{n}\right)^{\alpha / 2}\left(1+\cos \frac{u}{n}\right)^{\beta / 2}\left(\sin \frac{u}{n}\right)^{1 / 2} u^{-1 / 2} n^{-1 / 2}
$$

for $0<u \leqq \pi n$. If $u>n \pi$ then $\theta_{n}^{(\alpha \beta)}(u)$ is defined to be 0 . For each $n=1,2, \cdots$ we define a mapping from $\boldsymbol{L}^{\wedge}$ to $\boldsymbol{H}^{\wedge}$ by the formula

$$
\chi_{n} f \cdot(u)=f\left(\cos \frac{u}{n}\right) \theta_{n}^{(\alpha, \beta)}(u) \quad 0 \leqq u \leqq n \pi .
$$

Note that $\chi_{n} f \cdot(u)$ is 0 for $u>n \pi$. A simple computation shows that the mapping $\chi_{n}$ is isometric and into. We further define

$$
\chi_{n}^{*} f \cdot(x)=f(n \arccos x)(n \arccos x)^{1 / 2}\left(1-x^{2}\right)^{-1 / 4} w_{\alpha, \beta}(x)^{-1 / 2} n^{1 / 2} .
$$

The mapping $\chi_{n}^{*}$ is a partial isometry of $\boldsymbol{H}^{\wedge}$ onto $\boldsymbol{L}^{\wedge}$. Specifically $\chi_{n}^{*}$ is isometric on $\chi_{n} \boldsymbol{L}^{\wedge}$ and zero on $\left(\chi_{n} \boldsymbol{L}^{\wedge}\right)^{\perp}$, the orthogonal complement of $\chi_{n} \boldsymbol{L}^{\wedge}$ in $\boldsymbol{H}^{\wedge}$. Note that $\chi_{n}^{*} \chi_{n}=I$ on $\boldsymbol{L}^{\wedge}$ and $\chi_{n} \chi_{n}^{*}=I$ on $\chi_{n} \boldsymbol{L}^{\wedge}$ and 0 on $\left(\chi_{n} L^{\wedge}\right)^{\perp}$.

We next introduce various operators on these Hilbert spaces.

a. $E_{n}$ is defined on $\boldsymbol{L}$ by the formula

$$
E_{n} f \cdot(k)=\left\{\begin{array}{cl}
f(k) & \text { if } 0 \leqq k \leqq n \\
0 & \text { if } k>n .
\end{array}\right.
$$

The following operators are defined by "transferring" $E_{n}$ :

$$
\begin{aligned}
& E_{n}^{\wedge} \text { on } \boldsymbol{L}^{\wedge} \text { defined by } E_{n}^{\wedge}=\phi E_{n} \phi^{-1} ; \\
& F_{n}^{\wedge} \text { on } \boldsymbol{H}^{\wedge} \text { defined by } F_{n}^{\wedge}=\chi_{n} E_{n}^{\wedge} \chi_{n}^{*} .
\end{aligned}
$$

b. $T^{\wedge}$ is defined on $\boldsymbol{L}^{\wedge}$ by

$$
T^{\wedge} f \cdot(x)=[t(1)-t(x)] f(x) .
$$

We set:

$$
\begin{aligned}
& T \text { on } \boldsymbol{L} \quad \text { defined by } T=\phi^{-1} T^{\wedge} \phi ; \\
& T_{n}^{\wedge} \text { on } \boldsymbol{H}^{\wedge} \text { defined by } T_{n}^{\wedge}=\chi_{n} T^{\wedge} \chi_{n}^{*} ; \\
& S_{n}^{\wedge} \text { on } \boldsymbol{H}^{\wedge} \text { defined by } S_{n}^{\wedge}=2^{\omega} n^{2 \omega} L\left(n^{-2}\right)^{-1} T_{n}^{\wedge} .
\end{aligned}
$$

c. $S^{\wedge}$ is defined on $\boldsymbol{H}^{\wedge}$ by

$$
S^{\wedge} f \cdot(u)=u^{2 \omega} f(u) .
$$

d. $\boldsymbol{F}$ is defined on $\boldsymbol{H}$ by 


$$
F f \cdot(z)=\left\{\begin{array}{lr}
f(z) & 0 \leqq z \leqq 1 \\
0 & z>1
\end{array}\right.
$$

We set

$$
F^{\wedge} \text { on } \boldsymbol{H} \text { defined by } F^{\wedge}=\psi F \psi^{-1} \text {. }
$$

If $\lambda_{n, 1} \geqq \cdots \geqq \lambda_{n, n+1}$ are the eigen values of $C_{n}$, see $\S 1$, then

$$
t(1)-\lambda_{n, 1} \leqq \cdots \leqq t(1)-\lambda_{n, n+1}
$$

are the eigen values of the following operators:

$$
\begin{gathered}
E_{n} T E_{n} \mid E_{n} \boldsymbol{L}, \\
E_{n}^{\wedge} T^{\wedge} E_{n}^{\wedge} \mid E_{n}^{\wedge} \boldsymbol{L}^{\wedge}, \\
F_{n}^{\wedge} T_{n}^{\wedge} F_{n}^{\wedge} \mid F_{n}^{\wedge} \boldsymbol{H}^{\wedge},
\end{gathered}
$$

where these symbols are to be read " $E_{n} T E_{n}$ restricted to $E_{n} L$ ", etc. The eigen values of

$$
F_{n}^{\wedge} S_{n}^{\wedge} F_{n}^{\wedge} \mid F_{n}^{\wedge} H^{\wedge}
$$

are in increasing order $\left\{\left(t(1)-\lambda_{n, k}\right) 2^{\omega} n^{2 \omega} L\left(n^{-2}\right)^{-1}\right\}_{k=1}^{n+1}$. In the following sections we will show that $F_{n}^{\wedge} S_{n}^{\wedge} F_{n}^{\wedge}$ "converges" to $S^{\wedge} F^{\wedge}$ as $n \rightarrow \infty$, and thus, using the results of sections $2-7$, that if

$$
0<\mu_{1} \leqq \mu_{2} \leqq \cdots, \lim _{k \rightarrow \infty} \mu_{k}=+\infty
$$

are the eigenvalues of

$$
\left.S^{\wedge} F^{\wedge}\right|_{F^{\wedge} H^{\wedge}}
$$

then

$$
\lim _{n \rightarrow \infty}\left(t(1)-\lambda_{n, k}\right) 2^{\omega} n^{2 \omega} L\left(n^{-2}\right)^{-1}=\mu_{k} \quad k=1,2, \cdots,
$$

or equivalently

$$
\lambda_{n, k}=t(1)-\mu_{k} 2^{-\omega} n^{-2 \omega} L\left(n^{-2}\right)+o\left[n^{-2 \omega} L\left(n^{-2}\right)\right] .
$$

9. Convergence of $\left(S_{n}^{\wedge}\right)^{1 / 2}$ to $\left(S^{\wedge}\right)^{1 / 2}$. It follows from $\S 8$ that for every $f \in \boldsymbol{H}^{\wedge}$ we have

$$
T_{n}^{\wedge} f \cdot(u)=t_{n}(u) f(u) \quad 0 \leqq u<\infty,
$$

where 


$$
t_{n}(u)=\left\{\begin{array}{cr}
t(1)-t\left(\cos \frac{u}{n}\right) & 0 \leqq u \leqq n \pi \\
0 & n \pi<u .
\end{array}\right.
$$

Consequently

$$
S_{n}^{\wedge} f(u)=s_{n}(u) f(u)
$$

where

$$
s_{n}(u)=2^{\omega} n^{2 \omega}\left[L\left(n^{-2}\right)\right]^{-1} t_{n}(u)
$$

LEMMA 9a. Under the assumption of $\S 8$ we have

$$
\lim _{n \rightarrow \infty} s_{n}(u)=u^{2 \omega} \quad 0 \leqq u<\infty,
$$

and for any $\varepsilon>0$ there is a constant $M(\varepsilon)$ such that for $n=1,2, \ldots$

$$
0 \leqq s_{n}(u) \leqq M(\varepsilon)\left\{u^{\varepsilon}+u^{-\varepsilon}\right\} u^{2 \omega}, \quad 0 \leqq u<\infty .
$$

Proof. By assumption

$$
t(1)-t(x)=(1-x)^{\omega} L(1-x) \Lambda(x) \quad \text { as } x \rightarrow 1-
$$

where $L(y)$, continuous and positive for $0<y \leqq 2$, is slowly oscillating as $y \rightarrow 0+$, and where $\Lambda(1-)=1$. It is well known and easily verified that this implies that if $0<y_{1}, 0<y_{2}$, and $0<a_{1} \leqq y_{1} / y_{2} \leqq a_{2}$ then

$$
L\left(y_{1}\right) / L\left(y_{2}\right) \rightarrow 1 \quad \text { as } y_{1} \text { and } y_{2} \rightarrow 0 \text {. }
$$

We have

$$
s_{n}(u)=\left(2 n \sin \frac{u}{2 n}\right)^{2 \omega}\left[L\left(2 \sin ^{2} \frac{u}{2 n}\right) / L\left(n^{-2}\right)\right] \Lambda\left(\cos \frac{u}{n}\right)
$$

for $0 \leqq u \leqq n \pi$, and (1) is an immediate consequence of this formula.

From the fact that $L(y)$ is slowly oscillating as $y \rightarrow 0+$ it is easily verified that for each $\varepsilon>0$ there is a constant $A(\varepsilon)$ such that if $0<y_{1} \leqq 2,0<y_{2} \leqq 2$ then

$$
L\left(y_{1}\right) / L\left(y_{2}\right) \leqq A(\varepsilon)\left[\left(y_{1} / y_{2}\right)^{\varepsilon}+\left(y_{1} / y_{2}\right)^{-\varepsilon}\right] \text {. }
$$

It follows from (3) that if $M$ is sufficiently large then

$$
0 \leqq t(1)-t(x) \leqq M(1-x)^{\omega} L(1-x) \quad-1 \leqq x \leqq 1 .
$$

Consequently if $0 \leqq u \leqq n \pi$ we have 


$$
\begin{aligned}
& 0 \leqq s_{n}(u) \leqq M\left(2 n \sin \frac{u}{2 n}\right)^{2 \omega}\left[L\left(2 \sin ^{2} \frac{u}{2 n}\right) / L\left(n^{-2}\right)\right], \\
& 0 \leqq s_{n}(u) \leqq A(\varepsilon) M u^{2 \omega}\left[\left(2 n^{2} \sin ^{2} \frac{u}{2 n}\right)^{\varepsilon}+\left(2 n^{2} \sin ^{2} \frac{u}{2 n}\right)^{-\varepsilon}\right],
\end{aligned}
$$

from which (2) follows.

THEOREM $9 \mathrm{~b} . \quad\left(S^{\wedge}\right)^{1 / 2}$ is the closure of the strong limit of $\left(S_{n}^{\wedge}\right)^{1 / 2}$ as $n \rightarrow \infty$.

Proof. Let $f \in D\left[\left(S^{\wedge}\right)^{1 / 2}\right]$ and $\varepsilon>0$ be given. Let $f_{\delta}(u)=e^{-\delta u} f(u)$. It is evident that if $\delta$ is sufficiently small then

$$
\left\|f-f_{\delta}\right\|_{\boldsymbol{H}^{\wedge}}=\left\{\int_{0}^{\infty}|f(u)|^{2}\left|1-e^{-\delta u}\right|^{2} u d u\right\}^{1 / 2}<\varepsilon,
$$

and

$$
\left\|\left(S^{\wedge}\right)^{1 / 2}\left(f-f_{\delta}\right)\right\|_{\boldsymbol{H}^{\wedge}}=\left\{\int_{0}^{\infty}|f(u)|^{2}\left|1-e^{-\delta u}\right|^{2} u^{2 \omega+1} d u\right\}^{1 / 2}<\varepsilon .
$$

Moreover using (1) and (2) and the Lebesgue limit theorem it is evident that

$$
\left(S_{n}^{\wedge}\right)^{1 / 2} f_{\delta} \rightarrow\left(S^{\wedge}\right)^{1 / 2} f_{\delta} \quad \text { in } \boldsymbol{H}^{\wedge} \text { as } n \rightarrow \infty
$$

10. Convergence of $F_{n}^{\wedge}$ to $F^{\wedge}$.

TheOREM 10a. If $F_{n}^{\wedge}$ and $F^{\wedge}$ are defined as in $\S 8$ then $F_{n}^{\wedge}$ converges strongly to $F^{\wedge}$ as $n \rightarrow \infty$.

Proof. In order to shorten our formulas let us set

$$
R(k, n, u)=h_{k}^{-1 / 2} P_{k}^{(\alpha \beta)}\left(\cos \frac{u}{n}\right)\left\{w\left(\cos \frac{u}{n}\right)\right\}^{1 / 2}\left\{\sin \frac{u}{n}\right\}^{1 / 2} u^{-1 / 2}
$$

Starting from the definition of $F_{n}^{\wedge}$ as $\chi_{n} E_{n}^{\wedge} \chi_{n}^{*}$ it is easy to verify that for all $f \in H^{\wedge}$

$$
F_{n}^{\wedge} f \cdot(u)=\frac{1}{n} \sum_{k=0}^{n} R(k, n, u) a(k, n)
$$

if $0 \leqq u \leqq n \pi$ and $F_{n}^{\wedge} f \cdot(u)=0$ if $u>n \pi$, where

$$
a(k, n)=\int_{0}^{n x} f(\zeta) R(k, n, \zeta) \zeta d \zeta .
$$

Let us now assume that $f(u)$ is continuous for $0 \leqq u<\infty$ and vanishes except for $0<a_{1} \leqq u \leqq a_{2}<\infty$. We will show that under 
this assumption $F_{n}^{\wedge} f \cdot(u) \rightarrow F^{\wedge} f \cdot(u)$ uniformly in any subset $0<b_{1} \leqq$ $u \leqq b_{2} \leqq \infty$. We first note that

$$
\lim _{k \rightarrow \infty}\left(2^{\alpha+\beta} h_{k}^{-1}\right) / k=1 .
$$

This follows immediately from the formula of $\S 1$ defining $h_{k}$. Formula (5) of the Appendix asserts that

$$
\lim _{k \rightarrow \infty} k^{-\alpha} P_{k}^{(\alpha, \beta)}\left(\cos \frac{z}{k}\right)=(z / 2)^{-\alpha} J_{\alpha}(z)
$$

uniformly for $z$ in any compact subset of the complex plane. It is easily deduced from this that there exists a constant $M$ such that

$$
|R(k, n, \zeta)| \leqq M\left(\frac{k+1}{n}\right)^{a+(1 / 2)}
$$

if

$$
0<a_{1} \leqq \zeta \leqq a_{2}<\infty, \quad 0 \leqq k \leqq n, \quad n=1,2, \cdots
$$

Let us set

$$
\begin{aligned}
& \sum_{1}(\delta, n, u)=\frac{1}{n} \sum_{0 \leqq k<n \delta} R(k, n, u) a(k, n), \\
& \sum_{2}(\delta, n, u)=\frac{1}{n} \sum_{n \delta \leqq k \leqq n} R(k, n, u) a(k, n) .
\end{aligned}
$$

Then $F_{n}^{\wedge} f \cdot(u)=\sum_{1}(\delta, n, u)+\sum_{2}(\delta, n, u)$. Using (2), the inequality (4), and the corresponding inequality for $R(k, n, u)$ when $0<b_{1} \leqq$ $u \leqq b_{2}<\infty$ we find that

$$
\begin{aligned}
\left|\sum_{1}(\delta, n, u)\right| & \leqq M n^{-2-2 \alpha} \sum_{0 \leqq k<n \delta}(k+1)^{2 \alpha+1} \\
& \leqq M \delta^{2 \alpha+2}
\end{aligned}
$$

for

$$
b_{1} \leqq u \leqq b_{2}, \quad 0<\delta<1, \quad n=1,2, \cdots .
$$

It follows from (3) that

$$
\lim _{n \rightarrow \infty}\left[R(k, n, \zeta)-\left(\frac{k}{n}\right)^{1 / 2} J_{\alpha}\left(k_{\zeta}^{\zeta} n^{-1}\right)\right]=0
$$

uniformly for

$$
n \delta \leqq k \leqq n, \quad a_{1} \leqq \zeta \leqq a_{2} .
$$

Consequently

$$
\lim _{n \rightarrow \infty}\left[a(k, n)-g\left(k n^{-1}\right)(k / n)^{1 / 2}\right]=0
$$


where

$$
g\left(k n^{-1}\right)=\int_{0}^{\infty} f(\zeta) J_{\alpha}\left(k \zeta n^{-1}\right) \zeta d \zeta,
$$

uniformly in $k$ for $n \delta \leqq k \leqq n$. Here of course $g=\psi^{-1} f$. Using (5) again we have

$$
\lim _{n \rightarrow \infty}\left[R(k, n, u)-\left(\frac{k}{n}\right)^{1 / 2} J_{\alpha}\left(k u n^{-1}\right)\right]=0
$$

uniformly for

$$
n \delta \leqq k \leqq n \quad b_{1} \leqq u \leqq b_{2} .
$$

It now follows that

$$
\lim _{n \rightarrow \infty}\left|\sum_{2}(\delta, n, u)-\frac{1}{n} \sum_{n \delta \leqq k \leqq n} g\left(k n^{-1}\right) J_{\alpha}\left(k n^{-1} u\right) \frac{k}{n}\right|=0
$$

uniformly for $b_{1} \leqq u \leqq b_{2}$. We assert that

$$
\lim _{n \rightarrow \infty} \frac{1}{n} \sum_{n \delta \leqq k \leqq n} g\left(k n^{-1}\right) J_{\alpha}\left(k n^{-1} u\right) \frac{k}{n}=\int_{\delta}^{1} g(z) J_{\alpha}(u z) z d z
$$

uniformly for $b_{1} \leqq u \leqq b_{2}$. Indeed the sum on the left is a Riemann sum for the integral on the right, so that (7) is certainly true for each $u>0$. To show that it holds uniformly for $b_{1} \leqq u \leqq b_{2}$ it is sufficient to note that

$$
\left|\frac{d}{d u} \frac{1}{n} \sum_{n \delta \leqq k \leqq n} g\left(k^{-1} n\right) J_{\alpha}\left(k n^{-1} u\right) \frac{k}{n}\right| \leqq M
$$

uniformly for $b_{1} \leqq u \leqq b_{2} n=1,2, \cdots$ so that the sequence of functions on the left in (7) is equicontinuous. Given $\varepsilon>0$ let us choose $\delta>0$ so small that

$$
\left|\sum_{1}(\delta, n, u)\right|<\varepsilon / 2, \quad\left|\int_{0}^{\delta} g(z) J_{w}(u z) z d z\right|<\varepsilon / 2,
$$

for $b_{1} \leqq u \leqq b_{2}$. It then follows on collecting our estimates that $\left|F_{n}^{\wedge} f \cdot(u)-F^{\wedge} f(u)\right|<\varepsilon$ for $b_{1} \leqq u \leqq b_{2}$ and for all sufficiently large $n$.

Let $\boldsymbol{C}^{\wedge}$ be the set of functions $f \in \boldsymbol{H}^{\wedge}$ which are continuous and have support in $a_{1} \leqq u \leqq a_{2}$ for some $0<a_{1}<a_{2}<\infty$. Using what we have proved above and the fact that $\left\|F_{n}^{\wedge}\right\|=1 \quad n=1,2, \cdots$, we see that if $f \in C^{\wedge}$ then $F_{n}^{\wedge} f \rightarrow F^{\wedge} f$ as $n \rightarrow \infty$. Since $C^{\wedge}$ is dense in $\boldsymbol{H}^{\wedge}$ we see, again using the fact that $\left\|F_{n}^{\wedge}\right\|=1 n=1,2, \cdots$, that $F_{n}^{\wedge} \rightarrow F$ as $n \rightarrow \infty$. However weak convergence for projections implies strong convergence so that $F_{n}^{\wedge} \rightarrow F^{\wedge}$ as $n \rightarrow \infty$.

11. Convergence of $\left(S_{n}^{\wedge}\right)^{1 / 2} F_{n}^{\wedge}$ to $\left(S^{\wedge}\right)^{1 / 2} F$ Part I. It remains to 
prove that $\left(S^{\wedge}\right)^{1 / 2} F^{\wedge}$ is the closure of the strong limit of $\left(S_{n}^{\wedge}\right)^{1 / 2} F_{n}^{\wedge}$. The considerations here are considerably more involved than those of $\S \S 9$ and 10 and will occupy $\$ \S 11-13$.

Let $\boldsymbol{D}$ be the set of functions $h(z)$ in $\boldsymbol{H}$ which can be written in the form $h(z)=z^{\alpha} h_{1}(z)$ where $h_{1}(z)$ defined for $-\infty<z<\infty$ is even, infinitely differentiable and has compact support. We set $\boldsymbol{D}^{\wedge}=\psi \boldsymbol{D}$.

LEMMA 11a. If $f \in \boldsymbol{D}^{\wedge}$ then $f(u)=u^{\alpha} f_{1}(u)$ where $f_{1}(u)$ is the restriction to $0<u<\infty$ of an even continuous function satisfying $f_{1}(u)=0\left(u^{-r}\right)$ as $u \rightarrow+\infty$ for every $r$.

Proof. Suppose that $f \in \boldsymbol{D}^{\wedge}$ then, with an evident notation,

$$
f_{1}(u)=\int_{0}^{\infty} h_{1}(z) \Im_{\alpha}(z u) z^{2 \alpha+1} d z
$$

where $\Im_{\alpha}(z)=z^{-\alpha} J_{\alpha}(z)$ is an even continuous function satisfying $\left|\mathfrak{\Im}_{\alpha}(z)\right| \leqq A\left(1+|z|^{q}\right)$ for $0 \leqq z \leqq \infty$. Here $q=\max [0,-\alpha-(1 / 2)]$. If we set

$$
\Delta k \cdot(z)=k^{\prime \prime}(z)+\frac{2 \alpha+1}{z} k^{\prime}(z),
$$

then

$$
\Delta \Im_{\alpha}(z u)=-u^{2} \Im_{\alpha}(z u)
$$

Consequently

$$
\begin{aligned}
\left(-u^{2}\right)^{m} f_{1}(u) & =\int_{0}^{\infty} h_{1}(z)\left\{\left(-u^{2}\right)^{m} \Im_{\alpha}(u z)\right\} z^{2 \alpha+1} d z, \\
& =\int_{0}^{\infty} h_{1}(z)\left\{\Delta^{m} \Im_{\alpha}(u z)\right\} z^{2 \alpha+1} d z, \\
& =\int_{0}^{\infty}\left\{\Delta^{m} h_{1}(z)\right\} \Im_{\alpha}(u z) z^{2 \alpha+1} d z,
\end{aligned}
$$

where in the last step we have integrated by parts repeatedly. It is easy to deduce our assertion from this last formula.

Consider the rectangle

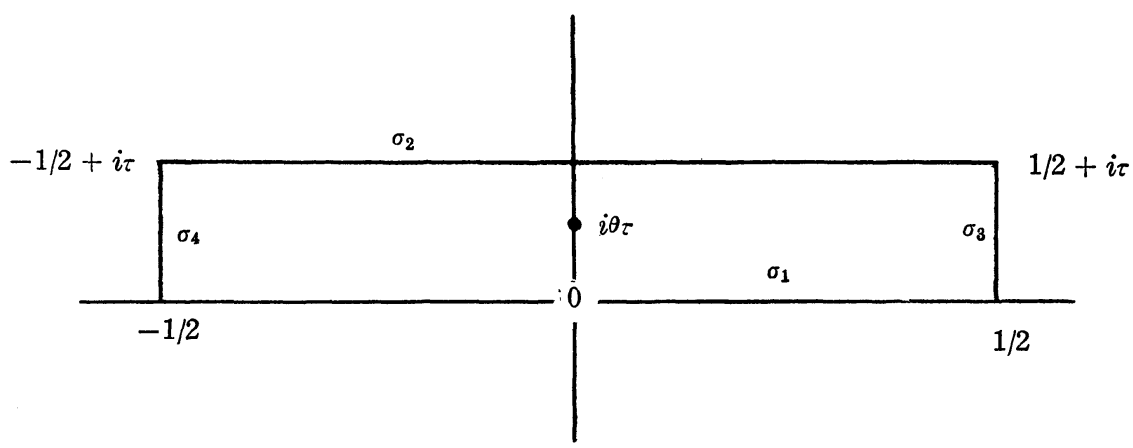


Let $\gamma_{k}(\theta)$ be the harmonic measure of the side $\sigma_{k}$ with respect to the point $i \theta \tau$. Later in this section we will need estimates of the $\gamma_{k}(\theta)$ 's.

LEMMA 11b. With the above notations we have the inequalities:

$$
\begin{aligned}
& \gamma_{1}(\theta) \geqq 1-\theta-2 \theta \tau \cosh \tau \pi, \\
& \gamma_{2}(\theta) \leqq \theta \\
& \gamma_{3}(\theta) \leqq \tau \theta \cosh \tau \pi, \\
& \gamma_{4}(\theta) \leqq \tau \theta \cosh \tau \pi .
\end{aligned}
$$

Proof. For the definition of harmonic measure and its basic properties see [8]. By the principle of domain extension $\gamma_{2}(\theta)$ is less than or equal to the harmonic measure of the line segment connection $-(1 / 2)+i \tau$ to $(1 / 2)+i \tau$ in the strip bounded by the lines $\operatorname{Im} z=\tau$ and $\operatorname{Im} z=0$

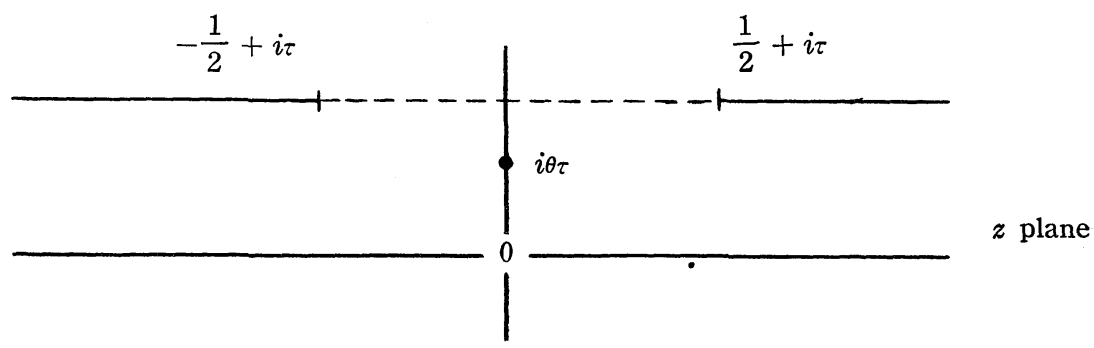

This is trivially less than the harmonic measure of the whole line $\operatorname{Im} z=\tau$. But this last is $\theta$, and thus $\gamma_{2}(\theta) \leqq \theta$.

A second application of the principle of domain extension shows that $\gamma_{3}(\theta)+\gamma_{4}(\theta)$ is less than the harmonic measure of the segments connecting $-1 / 2$ to $-(1 / 2)+i \tau$ and $1 / 2$ to $(1 / 2)+i \tau$ in the half strip bounded by the lines $R l z=-1 / 2, R l z=1 / 2$, and $\operatorname{Im} z=0$ and lying in the upper half plane.

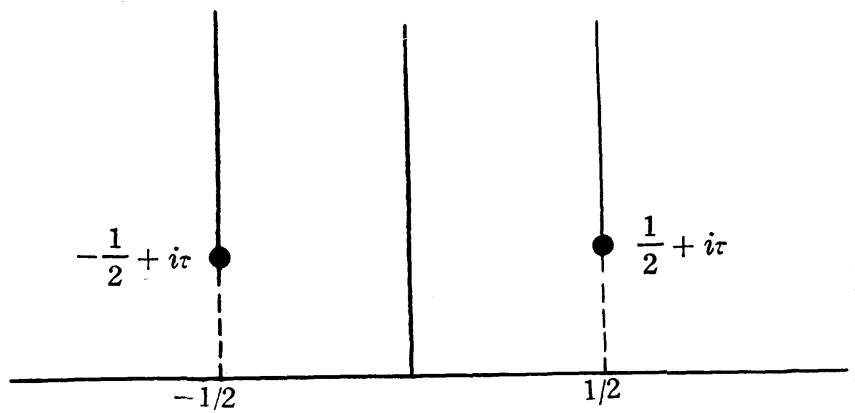

This is trivially less than the harmonic measure of the two vertical bounding lines. If $w=\sin \pi z$ then the half strip is mapped conformally 


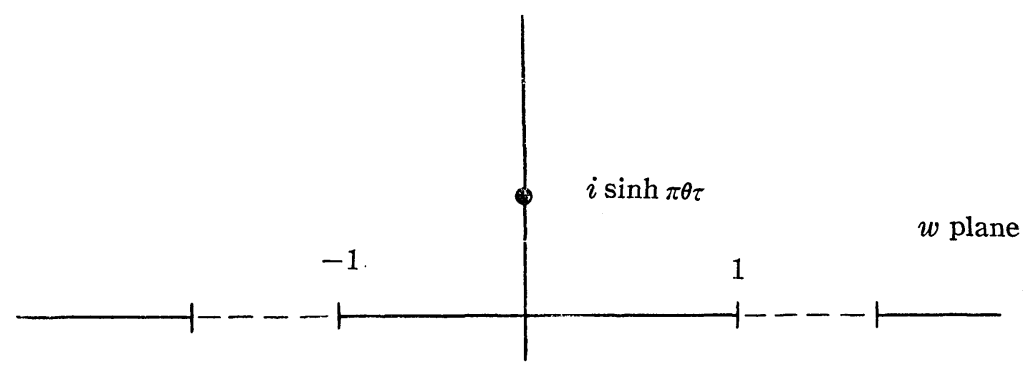

onto the half plane $\operatorname{Im} w \geqq 0$. The point $i \theta \tau$ goes over into $i \sinh \pi \theta \tau$. Since harmonic measures are invarient under conformal mapping we see that $\gamma_{3}(\theta)+\gamma_{4}(\theta)$ is less than the harmonic measure $\gamma$ of the infinte intervals $(\infty,-1]$ and $[1, \infty)$ with respect to $i \sinh \pi \theta \tau$. But this can be exactly computed using the Poisson formula for the half-plane. We find that

$$
\begin{aligned}
\gamma & \leqq \frac{2}{\pi} \sinh \pi \tau \theta \int_{1}^{\infty}\left[x^{2}+\sinh ^{2} \pi \tau \theta\right]^{-1} d x, \\
& \leqq \frac{2}{\pi} \sinh \pi \tau \theta .
\end{aligned}
$$

Since $\gamma_{3}(\theta)=\gamma_{4}(\theta)$ by symmetry and since $\gamma_{3}(\theta)+\gamma_{4}(\theta)<\gamma$ we find that $\gamma_{3}(\theta)$ and $\gamma_{4}(\theta)$ are both less than $(1 / \pi) \sinh \pi \tau \theta$. Using the mean value theorem we see that $(1 / \pi) \sinh \pi \tau \theta \leqq \tau \theta \cosh \pi \tau$, etc.

Let $\boldsymbol{D}_{1}$ be the subset of $\boldsymbol{D}$ consisting of those functions in $\boldsymbol{D}$ which vanish for $c_{1} \leqq z<\infty$ for some $c_{1}<1$, and let $\boldsymbol{D}_{1}^{\wedge}=\psi \boldsymbol{D}_{1}$. Let $\boldsymbol{D}_{2}$ be the subset of $\boldsymbol{D}$ consisting of those functions in $\boldsymbol{D}$ which vanish for $0 \leqq z \leqq c_{2}$ for some $c_{2}>1$ and let $\boldsymbol{D}_{2}^{\wedge}=\psi \boldsymbol{D}_{2}$. The principal result of the present section is the following.

Theorem 11c. If $f \in \boldsymbol{D}_{1}^{\wedge}$ or $\boldsymbol{D}_{2}^{\wedge}$ and if (as in $\S 10$ )

$$
a(k, n)=\int_{0}^{n \pi} f(\zeta) R(k, n, \zeta) \zeta d \zeta
$$

then for $\nu$ fixed $\nu=0, \pm 1, \pm 2, \cdots$, we have

$$
a(n, n+\nu)=0\left(n^{-r}\right)
$$

as $n \rightarrow \infty$

for every $r$.

Proof. We first consider the case $f \in \boldsymbol{D}_{1}^{\wedge}$ or $\boldsymbol{D}_{2}^{\wedge}$. We have

$$
a(k, n)=a_{1}(k, n)+a_{2}(k, n)
$$

where

$$
\begin{aligned}
& a_{1}(k, n)=\int_{0}^{n \pi / 2} f(\zeta) R(k, n, \zeta) \zeta d \zeta \\
& a_{2}(k, n)=\int_{n \pi / 2}^{n x} f(\zeta) R(k, n, \zeta) \zeta d \zeta .
\end{aligned}
$$


Using Lemma 11a and the relation

$$
\int_{0}^{n \pi} R(k, n, \zeta)^{2} \zeta d \zeta=n
$$

it is apparent that $a_{2}(n, k)=0\left(n^{-r}\right)$ as $n \rightarrow \infty$ for every $r>0$. Suppose now $f \in \boldsymbol{D}_{1}^{\wedge}$. If we set

$$
\begin{aligned}
& a_{1}^{+}(k, n)=\int_{0}^{n \pi / 2} Q_{k}^{+}\left(\cos \frac{\zeta}{n}\right) \Theta_{n}(\zeta) f_{1}(\zeta) d \zeta, \\
& a_{1}^{-}(k, n)=\int_{0}^{n \pi / 2} Q_{k}^{-}\left(\cos \frac{\zeta}{n}\right) \Theta_{n}(\zeta) f_{1}(\zeta) d \zeta,
\end{aligned}
$$

where

$$
\Theta_{n}(\xi)=h_{k}^{-1 / 2}\left\{w\left(\cos \frac{\zeta}{n}\right)\right\}^{-1 / 2}\left\{\sin \frac{\zeta}{n}\right\}^{1 / 2} \zeta^{\alpha+(1 / 2)},
$$

and where $f_{1}(\zeta)=\zeta^{-a} f(\zeta)$ then, see (10) of the Appendix,

$$
\pi i a_{1}(k, n)=a_{1}^{-}(k, n)-a_{1}^{+}(k, n) .
$$

Note that if $\beta$ is large $Q_{k}^{ \pm}(\cos \zeta / n) f_{1}(\zeta) \Theta_{n}(\zeta)$ may not be integrable near $\zeta=n \pi$. This is the reason for splitting off $a_{2}(n, k)$. Apply Cauchy's theorem to teach of the curves below and then let $\rho \rightarrow 0+$.

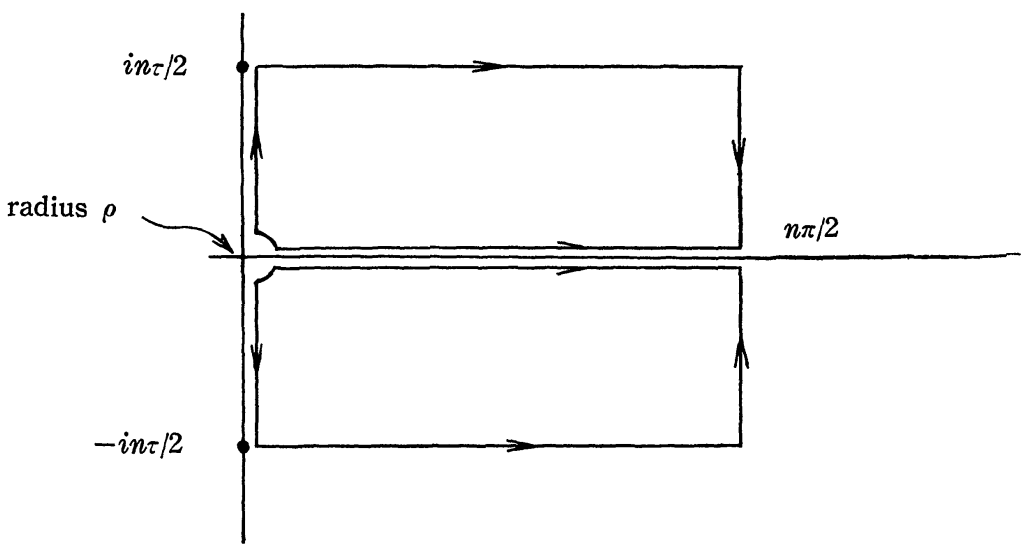

We obtain

$$
a_{1}^{ \pm}(k, n)=I_{1}^{ \pm}+I_{2}^{ \pm}+I_{3}^{ \pm}
$$

where

$$
\begin{array}{lll}
I_{1}^{-}=\int_{0}^{i n \tau / 2}, & I_{2}^{-}=\int_{i n \tau / 2}^{(i n \tau / 2)+(n \pi / 2)}, & I_{3}^{-}=\int_{(i n \tau / 2)+(n \pi / 2)}^{n \pi / 2}, \\
I_{1}^{+}=\int_{0}^{-i n \tau / 2}, & I_{2}^{+}=\int_{-i n \tau / 2}^{(-i n \tau / 2)+(n \pi / 2)}, & I_{3}^{+}=\int_{(-i n \tau / 2)+(n \pi / 2)}^{n \pi / 2},
\end{array}
$$


In all cases the integrand is

$$
f_{1}(\zeta) Q_{k}\left(\cos \frac{\zeta}{n}\right) \Theta_{n}(\zeta) d \zeta
$$

Let us put

$$
\Theta_{n}^{\sharp}(t)=h_{k}^{-1 / 2}\left\{\cosh \frac{t}{n}-1\right\}^{-\alpha / 2}\left\{\cosh \frac{t}{n}+1\right\}^{-\beta / 2}\left\{t \sinh \frac{t}{n}\right\}^{1 / 2} t^{\alpha}
$$

for $0<t<\infty$. Keeping careful track of arguments we find that

$$
\begin{aligned}
& I_{1}^{-}=-\int_{0}^{n \tau / 2} f_{1}(i t) Q_{k}\left(\cosh \frac{t}{n}\right) \Theta_{n}^{\sharp}(t) d t, \\
& I_{1}^{+}=-\int_{0}^{n \tau / 2} f_{1}(-i t) Q_{k}\left(\cosh \frac{t}{n}\right) \Theta_{n}^{\sharp}(t) d t .
\end{aligned}
$$

Since $f_{1}(\zeta)$ is even $I_{1}^{-}-I_{1}^{+}=0$ and thus

$$
\pi i a_{1}(k, n)=I_{2}^{-}+I_{2}^{-}-I_{2}^{+}-I_{3}^{+} .
$$

If $h=\psi^{-1} f$ then we have

$$
f_{1}(\zeta)=\int_{0}^{c_{1}} h(z) z^{-\alpha} \zeta^{-\alpha} J_{\alpha}(z \zeta) z^{\alpha+1} d z
$$

where $c_{1}<1$. It follows that $f_{1}(\zeta)$ is an entire function of $\zeta$ and that for any $c, c_{1}<c<1$,

$$
\left|f_{1}\left(\xi+\frac{1}{2} i n \tau\right)\right| \leqq A e^{c \tau n / 2} \quad 0 \leqq \xi \leqq n \pi / 2 ;
$$

see $[1 ; p .85]$. By (11) of the Appendix we have

$$
\left|Q_{n+\nu}\left[\cos \left(\frac{1}{2} i \tau+\xi n^{-1}\right)\right]\right| \leqq A n^{q} e^{-\tau n / 2}, \quad 0 \leqq \xi \leqq n \pi / 2 .
$$

if $\nu$ is fixed. Since

$$
\left|\Theta_{n}^{\sharp}\left(\frac{1}{2} i \tau+\xi\right)\right| \leqq A n^{\alpha+1} \quad 0 \leqq \xi \leqq n \pi / 2
$$

we see that $I_{2}^{-}$vanishes exponentially as $n \rightarrow \infty . \quad I_{2}^{+}$can be similarly treated.

In order to estimate $I_{3}^{-}$we consider the rectangle below. 


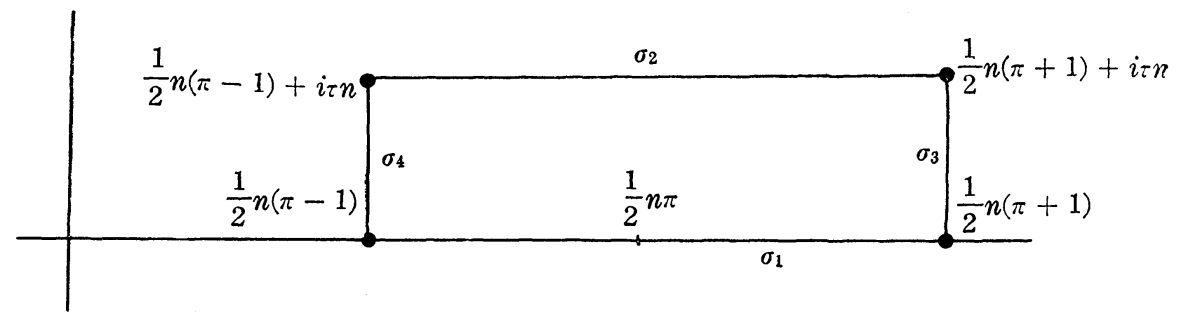

This rectangle is similar to the rectangle of Lemma 11b. Let

$$
M_{i}=\text { l.u.b. }\left|f_{1}(\zeta)\right| \zeta \text { on } \sigma_{i} \quad i=1,2,3,4 .
$$

By the principle of harmonic majoration

$$
\log \left|f_{1}\left(\frac{1}{2} n \pi+i n \tau \theta\right)\right| \leqq \sum_{i=1}^{4} \gamma_{i}(\theta) \log M_{i} .
$$

We have $M_{1} \leqq A n^{-r}, M_{i} \leqq A e^{c \tau n} i=2,3,4$. By Lemma $11 b$ if $\tau$ is sufficiently small

$$
\gamma_{1}(\theta) \geqq \frac{1}{3}, \quad c\left\{\gamma_{2}(\theta)+\gamma_{3}(\theta)+\gamma_{4}(\theta)\right\} \leqq \theta, \quad 0 \leqq \theta \leqq \frac{1}{2} .
$$

For $\tau$ so chosen

$$
\left|f_{1}\left(\frac{1}{2} n \pi+i \eta\right)\right| \leqq A e^{\eta} n^{-r / 3} \quad 0 \leqq \eta \leqq \tau n / 2,
$$

uniformly in $n$. On the other hand by (11) of the Appendix

$$
\left|Q_{n+\nu}\left(\cos \left(\frac{1}{2} \pi+i \eta n^{-1}\right)\right)\right| \leqq A n^{q} e^{-\eta} \quad 0 \leqq \eta \leqq \tau n / 2,
$$

and an elementary argument shows that

$$
\left|\Theta_{n}\left(\frac{1}{2} n \pi+i \eta\right)\right| \leqq A n^{\alpha} \quad 0 \leqq \eta \leqq \tau n / 2 .
$$

Since $r$ is arbitrary these estimates imply that $I_{3}^{-}=0\left(n^{-r}\right)$ as $n \rightarrow \infty$ for every $r . I_{3}^{+}$can be dealt with similarly.

We now turn to the case $f \in \boldsymbol{D}_{2}^{\wedge}$. We have, if $h=\psi^{-1} f$,

$$
f(\zeta)=\int_{c_{1}}^{c_{2}} h(z) J_{\alpha}(z \zeta) z d z
$$

where $1<c_{1}<c_{2}<\infty$. Since, see [1; p. 4],

$$
J_{a}(z)=\frac{1}{2} H_{\alpha}^{(1)}(z)+\frac{1}{2} H_{\alpha}^{(2)}(z)
$$

we have

$$
2 f(\zeta)=f^{(1)}(\zeta)+f^{(2)}(\zeta),
$$


where

$$
\begin{aligned}
& f^{(1)}(\zeta)=\int_{c_{1}}^{c_{2}} h(z) H_{\alpha}^{(1)}(z \zeta) z d z, \\
& f^{(2)}(\zeta)=\int_{c_{1}}^{c_{2}} h(z) H_{\alpha}^{(2)}(z \zeta) z d z
\end{aligned}
$$

and hence

$$
a_{1}(k, n)=a_{1}^{(1)}(k, n)+a_{1}^{(2)}(k, n)
$$

where

$$
a_{1}^{(i)}(k, n)=\int_{0}^{n \pi / 2} f^{(i)}(\zeta) R(k, n, \zeta) \zeta d \zeta, \quad i=1,2
$$

The functions $f^{(1)}(\zeta)$ and $f^{(2)}(\zeta)$ are analytic in the plane slit from $-\infty$ to 0 . Let us apply Cauchy's theorem to one or the other of the two curves below and then let $\rho \rightarrow 0+$.

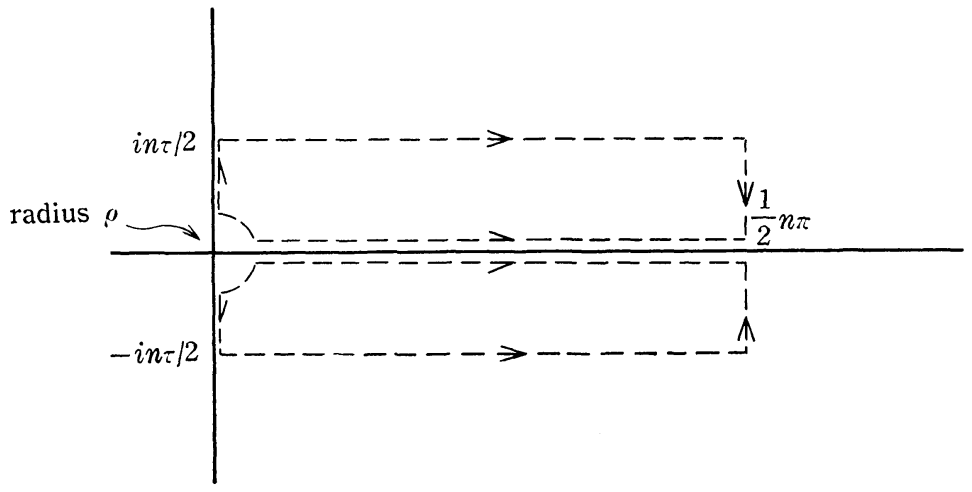

We see that

$$
\begin{array}{ccc}
a_{1}^{(i)}(k, n)=I_{1}^{(i)}+I_{2}^{(i)}+I_{3}^{(i)} & i=1,2 . \\
I_{1}^{(1)}=\int_{0}^{i n \tau / 2}, & I_{2}^{(1)}=\int_{i n \tau / 2}^{(i n \tau / 2)+(n \pi / 2)}, \quad I_{3}^{(1)}=\int_{(i n \tau / 2)+(n \pi / 2)}^{n \pi / 2}, \\
I_{1}^{(2)}=\int_{0}^{-i n \tau / 2}, & I_{2}^{(2)}=\int_{-i n \tau / 2}^{(-i n \tau / 2)+(n \pi / 2)}, & I_{3}^{(2)}=\int_{(-i n \tau / 2)+(n \pi / 2)}^{n \pi / 2} .
\end{array}
$$

In $I_{j}^{(i)}, j=1,2,3$ the integrand is

$$
f^{(i)}(\zeta) P_{k}^{(\alpha \beta)}\left(\cos \frac{\zeta}{n}\right) \Omega_{n}(\zeta) d \zeta
$$

where

$$
\Omega_{n}(\zeta)=h_{k}^{-1 / 2} w\left(\cos \frac{\zeta}{n}\right)^{1 / 2}\left(\sin \frac{\zeta}{n}\right)^{1 / 2} \zeta^{+1 / 2}
$$


From this we see that if

$$
\Omega_{n}^{*}(t)=h_{k}^{-1 / 2}\left(\cosh \frac{t}{n}-1\right)^{\alpha / 2}\left(\cosh \frac{t}{n}+1\right)^{\beta / 2}\left\{t \sinh \frac{t}{n}\right\}^{1 / 2}
$$

then

$$
\begin{aligned}
& I_{1}^{(1)}=-e^{\pi i \alpha / 2} \int_{0}^{\tau n / 2} f^{(1)}\left(t e^{\pi i / 2}\right) P_{k}^{(\alpha, \beta)}\left(\cosh \frac{t}{n}\right) \Omega_{n}^{\sharp}(t) d t, \\
& I_{1}^{(2)}=-e^{-\pi i \alpha / 2} \int_{0}^{\tau n / 2} f^{(2)}\left(t e^{-\pi i / 2}\right) P_{k}^{(\alpha \beta \beta)}\left(\cosh \frac{t}{n}\right) \Omega_{n}^{\sharp}(t) d t .
\end{aligned}
$$

Adding we find that

$$
\begin{aligned}
I_{1}^{(1)}+I_{1}^{(2)}=-\int_{0}^{\tau n / 2}\left[e^{\pi i \alpha / 2} f^{(1)}\left(t e^{\pi i / 2}\right)\right. & \left.+e^{-\pi i \alpha / 2} f^{(2)}\left(t e^{-\pi i / 2}\right)\right] \\
\cdot & P_{k}^{(\alpha \beta)}\left(\cosh \frac{t}{n}\right) \Omega_{n}^{\sharp}(t) d t .
\end{aligned}
$$

Since $\arg t=0$

$$
e^{\pi i \alpha / 2} H_{\alpha}^{(1)}\left(t e^{\pi i / 2}\right)+e^{-\pi i \alpha / 2} H_{\alpha}^{(2)}\left(t e^{-\pi i / 2}\right)=0,
$$

see $[1 ; \mathrm{p} .5]$. We have $I_{1}^{(1)}+I_{1}^{(2)}=0$ and thus

$$
a_{1}(n, k)=I_{2}^{(1)}+I_{3}^{(1)}+I_{2}^{(2)}+I_{3}^{(2)} \text {. }
$$

It follows from [1; p. 85] that if $1<c<c_{2}$ then

$$
\left|f^{(1)}\left(\frac{1}{2} i \tau n+\xi\right)\right| \leqq A e^{-c \tau n / 2} \quad 0 \leqq \xi \leqq n \pi / 2
$$

while by (8) of the Appendix

$$
\left|P_{n+\nu}\left(\cos \left[\frac{1}{2} i \tau+\xi n^{-1}\right]\right)\right| \leqq A n^{q} e^{\tau n / 2} \quad 0 \leqq \xi \leqq n \pi / 2 .
$$

Since trivially

$$
\left|\Omega_{n}\left(\frac{1}{2} i n \tau+\xi\right)\right| \leqq A n \quad 0 \leqq \xi \leqq n \pi / 2
$$

we see that $I_{2}^{(1)}$ vanishes exponentially as $n \rightarrow \infty$ and thus that $I_{2}^{(1)}=$ $0\left(n^{-r}\right)$ as $n \rightarrow \infty$ for every $r$. Similar considerations apply to $I_{2}^{(2)}$.

Using Lemmas $11 \mathrm{a}$ and $11 \mathrm{~b}$ we can deal with $I_{3}^{(1)}$ and $I_{3}^{(2)}$.very much in the way we dealt with $I_{3}^{+}$and $I_{3}^{-}$.

12. Convergence of $\left(S_{n}^{\wedge}\right)^{1 / 2} F_{n}^{\wedge}$ to $\left(S^{\wedge}\right)^{1 / 2} F^{\wedge}$. Part II.

Lemma 12a. Let $g \in \boldsymbol{D}_{1}^{\wedge}$. Then for every nonnegative integer $N$ 
we have

$$
\varlimsup_{n \rightarrow \infty} \int_{0}^{\infty} u^{4 N}\left|F_{n}^{\wedge} g \cdot(u)\right|^{2} u d u \leqq\left(\frac{\pi}{2}\right)^{4 N} \int_{0}^{\infty} u^{4 N}|g(u)|^{2} u d u
$$

Proof. By (1) of $\S 10$ we have,

$$
F_{n}^{\wedge} g \cdot(u)=n^{-1} \sum_{k=0}^{n} R(k, n, u) a(k, n)
$$

where

$$
a(k, n)=\int_{0}^{n \pi} R(k, n, u) g(u) u d u
$$

Let $g_{1}(u)=g(u)(1-\cos u / n)$. Using the recursion formula, (1) of the Appendix, a short computation shows that

$$
\begin{aligned}
(1 & \left.-\cos \frac{u}{n}\right) F_{n}^{\wedge} g \cdot(u)-{F_{n}^{\wedge}}_{1} g_{1}(u) \\
& =h_{n}^{1 / 2} h_{n+1}^{-1 / 2} C_{n+1} a(n+1, n) R(n, n, u)-h_{n+1}^{1 / 2} h_{n}^{-1 / 2} A_{n} a(n, n) R(n+1, n, u) .
\end{aligned}
$$

Using Theorem 11c this implies that

$$
\left\|\left(1-\cos \frac{u}{n}\right) F_{n}^{\wedge} g \cdot(u)-F_{n}^{\wedge} g_{1} \cdot(u)\right\|=0\left(n^{-r}\right)
$$

and thus since $\left\|\boldsymbol{F}_{n}^{\wedge}\right\|=1$

$$
\begin{aligned}
& \int_{0}^{n \pi}\left|1-\cos \frac{u}{n}\right|^{2}\left|F_{n}^{\wedge} g \cdot(u)\right|^{2} u d u \leqq \int_{0}^{n \pi}\left|F_{n}^{\wedge} g_{1} \cdot(u)\right|^{2} u d u+0\left(n^{-r}\right), \\
& \int_{0}^{n \pi}\left|1-\cos \frac{u}{n}\right|^{2}\left|F_{n}^{\wedge} g \cdot(u)\right|^{2} u d u \leqq \int_{0}^{\infty}\left|1-\cos \frac{u}{n}\right|^{2}|g(u)|^{2} u d u+0\left(n^{-r}\right) .
\end{aligned}
$$

Now

$$
1-\cos \frac{u}{n}=2 \sin ^{2}\left(\frac{u}{2 n}\right) \begin{cases}\geqq 2\left(\frac{2}{\pi} \frac{u}{2 n}\right)^{2} & 0 \leqq u \leqq n \pi \\ \leqq 2\left(\frac{u}{2 n}\right)^{2} & 0 \leqq u<\infty\end{cases}
$$

Multiplying (1) through by (1/4) $\pi^{4} n^{4}$ and using (2) we find that

$$
\int_{0}^{n \pi} u^{4}\left|F_{n}^{\wedge} g \cdot(u)\right|^{2} u d u \leqq\left(\frac{\pi}{2}\right)^{4} \int_{0}^{\infty} u^{4}|g(u)|^{2} u d u+0\left(n^{-r+2}\right),
$$

which implies our result for $N=1$. The argument however is valid in general if we use $(1-\cos u / n)^{N}$ in place of $(1-\cos u / n)$. 


$$
\lim _{n \rightarrow \infty}\left\|\left(S^{\wedge}\right)^{1 / 2} F^{\wedge} f-\left(S_{n}^{\wedge}\right)^{1 / 2} F_{n}^{\wedge} f\right\|=0 .
$$

Proof. An elementary argument gives

$$
\left\|\left(S^{\wedge}\right)^{1 / 2} F^{\wedge} f-\left(S_{n}^{\wedge}\right)^{1 / 2} F_{n}^{\wedge} f\right\|^{2} \leqq I_{1}+2 I_{2}+2 I_{3}
$$

where

$$
\begin{aligned}
& I_{1}=\int_{0}^{T}\left|u^{\omega} F^{\wedge} f \cdot(u)-s_{n}(u)^{1 / 2} F_{n}^{\wedge} f \cdot(u)\right|^{2} u d u, \\
& I_{2}=\int_{T}^{\infty} u^{2 \omega}\left|F^{\wedge} f \cdot(u)\right|^{2} u d u, \\
& I_{3}=\int_{T}^{\infty} s_{n}(u)\left|F_{n}^{\wedge} f \cdot(u)\right|^{2} u d u .
\end{aligned}
$$

By (2) of $\S 9$ (if $T \geqq 1$ ) then

$$
I_{3} \leqq A \int_{T}^{\infty} u^{2 \omega+1}\left|F_{n}^{\wedge} \cdot(u)\right|^{2} u d u .
$$

Choose $N$ so that if $a=4 N-(2 \omega+1)$ then $a>0$. We then have

$$
I_{3} \leqq A T^{-a} \int_{0}^{\infty} u^{4 N}\left|F_{n}^{\wedge} f \cdot(u)\right|^{2} u d u .
$$

It is now evident from Lemma $12 \mathrm{a}$ that if $T$ is sufficiently large then

$$
\varlimsup_{n \rightarrow \infty} I_{3}<\varepsilon / 4 \text {. }
$$

Since $F^{\wedge} f \cdot(u)=f(u)$ for $f \in \boldsymbol{D}_{1}^{\wedge}$ we see using Lemma 11a that for all sufficiently, large $T$

$$
I_{2}<\varepsilon / 4 \text {. }
$$

Suppose now that $T$ has been chosen so that (3) and (4) hold. Since $\lim _{n \rightarrow \infty} s_{n}(u)^{1 / 2}=u^{\omega}$ boundedly for $0 \leqq u \leqq T$ and since by Theorem $10 \mathrm{~b}$ $F_{n}^{\wedge} f \cdot(u) \rightarrow F^{\wedge} f \cdot(u)$ in $\boldsymbol{H}^{\wedge}$ we have

$$
\lim _{n \rightarrow \infty} I_{1}=0 \text {. }
$$

Combining (3), (4) and (5) gives

$$
\varlimsup_{n \rightarrow \infty}\left\|\left(S^{\wedge}\right)^{1 / 2} F^{\wedge} f-\left(S_{n}^{\wedge}\right)^{1 / 2} F_{n}^{\wedge} f\right\| \leqq \varepsilon,
$$

but $\varepsilon$ is arbitrary, etc.

LEMMA 12c. Let $g \in \widehat{D_{2}^{\wedge}}$; then for every nonnegative integer $N$ we have

$$
\varlimsup_{n \rightarrow \infty} \int_{0}^{\infty} u^{4 N}\left|F_{n}^{\wedge} g \cdot(u)\right|^{2} u d u \leqq\left(\frac{\pi}{2}\right)^{4 N} \int_{0}^{\infty} u^{4 N}|g(u)|^{2} u d u .
$$


THEOREM 12d. If $f \in \boldsymbol{D}_{2}^{\wedge}$ then

$$
\lim _{n \rightarrow \infty}\left\|\left(S^{\wedge}\right)^{1 / 2} F^{\wedge} f-\left(S_{n}^{\wedge}\right)^{1 / 2} F_{n}^{\wedge} f\right\|=0 .
$$

Note that for $f \in \boldsymbol{D}_{2}^{\wedge}, F^{\wedge} f=0$.

The demonstrations of Lemma $12 \mathrm{c}$ and Theorem $12 \mathrm{~d}$ are so much like those of Lemma $12 \mathrm{a}$ and Theorem $12 \mathrm{~b}$ that they are omitted.

13. Convergence of $\left(S_{n}^{\wedge}\right)^{1 / 2} F_{n}^{\wedge}$ to $\left(S^{\wedge}\right)^{1 / 2} F^{\wedge}$, Part III. If

$$
\begin{aligned}
& f(u)=\int_{0}^{\infty} f^{*}(z) J_{\alpha}(z u) z d z \\
& g(u)=\int_{0}^{\infty} g^{*}(z) J_{\alpha}(z u) z d z
\end{aligned}
$$

and if

$$
h^{*}(z)=\int_{0}^{\infty}\left\{f(u) g(u) u^{-\alpha}\right\} J_{\alpha}(u z) u d u
$$

then $h^{*}(z)$ is a "convolution" of $f^{*}(z)$ and $g^{*}(z)$. Indeed if $\alpha \geqq-1 / 2$ then there exists a very interesting formula for $h^{*}$ in terms of $f^{*}$ and $g^{*}$, and it is possible using this formula to read off simple properties concerning supports such as those proved below. See, for example, [3] or [4]. However these arguments are not available if $-1<\alpha<-1 / 2$.

Let $\delta(z)$ be a nonnegative function in $\boldsymbol{D}_{1}$ such that

$$
\int_{0}^{\infty} \delta(z) z^{\alpha+1} d z=2^{\alpha} \Gamma(\alpha+1) \quad \alpha>-1 .
$$

We define

$$
\Delta(u)=\int_{0}^{\infty} \delta(z) J_{a}(z u) z d z
$$

Let also $\Delta_{1}(u)=u^{-\infty} \Delta(u)$. We know from Lemma 11a that $\Delta_{1}(u)=$ $0\left(u^{-r}\right)$ as $u \rightarrow+\infty$ for every $r$. It is easily seen using $(1)$ that $\Delta_{1}(0)=1$. Also $A_{1}(u)$ is the restriction to the real axis of an even entire function $\Delta_{1}(w)$ which satisfies $\left|\Delta_{1}(w)\right| \leqq A e^{|v|}\left(1+|w|^{q}\right), \quad w=u+i v$, where $q=\max (0,-1 / 2-\alpha)$.

LemMA 13a. Let $f^{*}(z) \in \boldsymbol{H}$. If

1. $f^{*}(z)$ vanishes for $c \leqq z<\infty$;

2. $f(u)=\int_{0}^{\infty} f^{*}(z) J_{\alpha}(u z) z d z$;

3. $f^{*}(\lambda, z)=\int_{0}^{\infty} f(u) \Delta_{1}(\lambda u) J_{a}(u z) u d u$, 
then $z^{-\alpha} f^{*}(\lambda, z)$ is the restriction to $0 \leqq z<\infty$ of an even infinitely differentiable function and $f^{*}(\lambda, z)=0$ for $c+\lambda \leqq z<\infty$.

Proof. We will merely sketch the demonstration. Since

$$
J_{\alpha}(z)=\frac{1}{2}\left[H_{\alpha}^{(1)}(z)+H_{\alpha}^{(2)}(z)\right]
$$

we have

$$
2 f^{*}(\lambda, z)=\int_{0}^{\infty}\left[H_{\alpha}^{(1)}(z u)+H_{\alpha}^{(2)}(z u)\right] f_{1}(u) \Delta_{1}(\lambda u) u^{\alpha+1} d u .
$$

It is easily seen that this can be rewritten as

$$
2 f^{*}(\lambda, z)=\int_{-\infty}^{\infty} H_{\alpha}^{(1)}(z u) f_{1}(u) \Delta_{1}(\lambda u) u^{\alpha+1} d u
$$

where $z u$ has argument 0 for $0<u<\infty$ and argument $\pi$ for $-\infty<u<0$. By Cauchy's theorem if

$$
I=\int_{-T}^{T} H_{\alpha}^{(1)}(z u) f_{1}(u) \Delta_{1}(\lambda u) u^{\alpha+1} d u
$$

then

$$
I=I_{1}+I_{2}+I_{3}
$$

where

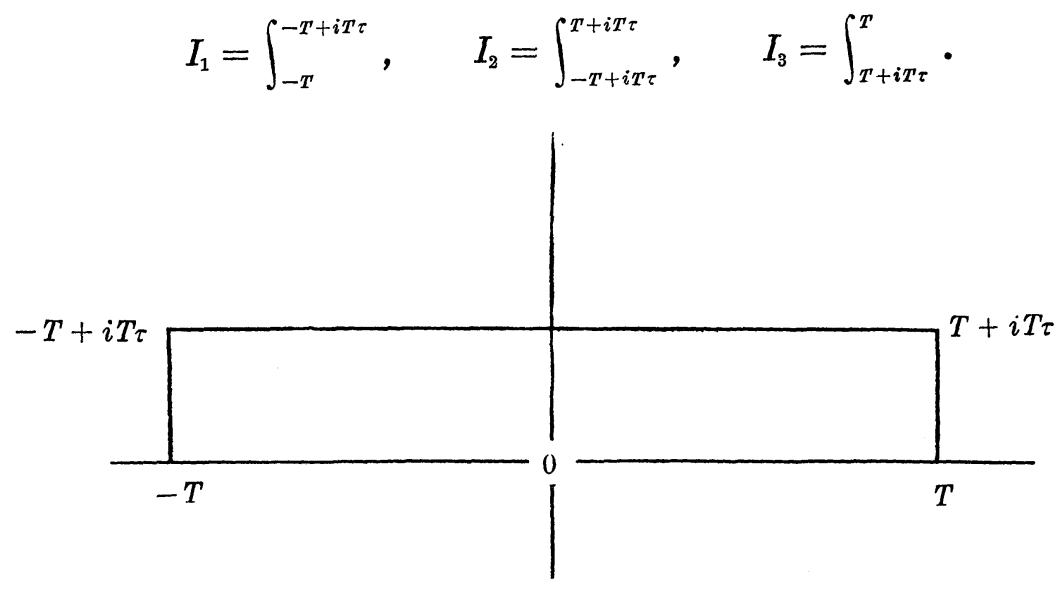

Fixing $\tau$ conveniently we can show by arguments like those in $\S 11$ that if $z>c+\lambda, I_{1}, I_{2}, I_{3} \rightarrow 0$ as $T \rightarrow \infty$. Using the fact that $f(u) \Delta_{1}(\lambda u)=0\left(u^{-r}\right)$ as $u \rightarrow \infty$ for every $r$ we see from the formula defining $f^{*}(\lambda, z)$ that $z^{-\alpha} f^{*}(\lambda, z)$ is the restriction to $0 \leqq z<\infty$ of an even infinitely differentiable function. By continuity $f^{*}(\lambda, z)=0$ 
for $z=c+\lambda$.

LEMMA 13b. Let $f^{*}(z) \in \boldsymbol{H}$. If

1. $f^{*}(z)$ vanishes for $0 \leqq z \leqq c, c>0$;

2. $f(u)=\int_{0}^{\infty} f^{*}(z) J_{\omega}(u z) z d z \quad\left(M_{2}\right)$;

3. $f^{*}(\lambda, u)=\int_{0}^{\infty} f(u) \Delta_{1}(\lambda u) J_{a}(u z) u d u$;

then $z^{-\alpha} f^{*}(\lambda, z)$ is the restriction to $0<z<\infty$ of an even infinitely differentiable function and $f^{*}(\lambda, z)=0$ for $0 \leqq z \leqq c-\lambda$ if $c-\lambda>0$.

Proof. Again we merely sketch the proof. We have

$$
2 f(u)=f^{(1)}(u)+f^{(2)}(u)
$$

where

$$
\begin{aligned}
& f^{(1)}(u)=\int_{0}^{\infty} H_{\alpha}^{(1)}(z u) f(z) z d z, \\
& f^{(2)}(u)=\int_{0}^{\infty} H_{\alpha}^{(2)}(z u) f(z) z d z,
\end{aligned}
$$

and thus

$$
2 f^{*}(\lambda, z)=\lim _{T \rightarrow \infty}\left[I^{(1)}+I^{(2)}\right]
$$

where

$$
\begin{aligned}
& I^{(1)}=\int_{0}^{T} f^{(1)}(u) \Delta_{1}(\lambda u) J_{\alpha}(u z) u d u \\
& I^{(2)}=\int_{0}^{T} f^{(2)}(u) \Delta_{1}(\lambda u) J_{\alpha}(u z) u d u .
\end{aligned}
$$

By Cauchy's theorem

$$
\begin{aligned}
& I^{(1)}=I_{1}^{(1)}+I_{2}^{(1)}+I_{3}^{(1)}, \\
& I^{(2)}=I_{1}^{(2)}+I_{2}^{(2)}+I_{3}^{(2)},
\end{aligned}
$$

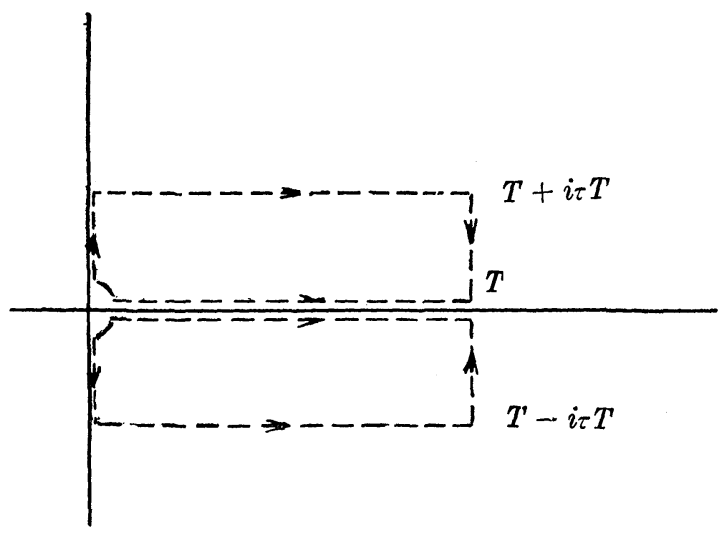


where

$$
\begin{array}{lll}
I_{1}^{(1)}=\int_{0}^{i \tau T}, & I_{2}^{(1)}=\int_{i \tau T}^{i \tau T+T}, & I_{3}^{(1)}=\int_{i \tau T+T}^{T}, \\
I_{1}^{(2)}=\int_{0}^{-i \tau T}, & I_{2}^{(2)}=\int_{-i \tau T}^{-i \tau T+T}, & I_{3}^{(2)}=\int_{-i \tau T+T}^{T} .
\end{array}
$$

It is easily verified that $I_{1}^{(1)}+I_{1}^{(2)}=0$, and arguments like those used in $\S 11$ suffice to prove that $I_{2}^{(1)}, I_{3}^{(1)}, I_{2}^{(2)}$, and $I_{3}^{(2)} \rightarrow 0$ as $T \rightarrow \infty$ if $\tau$ is suitably chosen, for $0 \leqq z<c-\lambda$, etc.

THEOREM 13c. Let $f \in D\left[\left(S^{\wedge}\right)^{1 / 2} F^{\wedge}\right]$; then given $\varepsilon>0$ there exists $h \in \boldsymbol{D}\left[\left(S^{\wedge}\right)^{1 / 2} F^{\wedge}\right]$ such that:

$$
\begin{gathered}
\|f-h\|<\varepsilon, \quad\left\|\left(S^{\wedge}\right)^{1 / 2} F^{\wedge}\{f-h\}\right\|<\varepsilon, \\
\left(S_{n}^{\wedge}\right)^{1 / 2} F_{n}^{\wedge} h \rightarrow\left(S^{\wedge}\right)^{1 / 2} F^{\wedge} h
\end{gathered}
$$$$
\text { as } n \rightarrow \infty \text {. }
$$

Proof. It is obviously sufficient to consider two cases. $F^{\wedge} f=f$ and $F^{\wedge} f=0$.

Suppose that $F^{\wedge} f=f$. By assumption $f \in \boldsymbol{D}\left[\left(S^{\wedge}\right)^{1 / 2} F^{\wedge}\right]$ so that

$$
\left\|\left(S^{\wedge}\right)^{1 / 2} F^{\wedge} f\right\|^{2}=\int_{0}^{\infty} u^{2 \omega}|f(u)|^{2} u d u<\infty .
$$

For $0<\theta<1$ let $g(u)=f(\theta u)$. Then if $f^{*}=\psi^{-1} f, g^{*}=\psi^{-1} g$ we have

$$
\begin{aligned}
g^{*}(z) & =\int_{0}^{\infty} f(\theta u) J_{\alpha}(u z) u d u \\
& =\int_{0}^{\infty} f(u) J_{\alpha}\left(u z \theta^{-1}\right) \theta^{-2} u d u \\
& =f^{*}\left(z \theta^{-1}\right) \theta^{-2} .
\end{aligned}
$$

$F^{\wedge} f=f$ implies that $f^{*}(z)=0$ for $z>1$. It follows that $g^{*}(z)=0$ for $z>\theta$. Consequently $F^{\wedge} g=g$ as well. It is also evident that $g \in \boldsymbol{D}\left[\left(S^{\wedge}\right)^{1 / 2} F^{\wedge}\right]$. Since

$$
\begin{gathered}
\|f-g\|^{2}=\int_{0}^{\infty}|f(u)-f(\theta u)|^{2} u d u, \\
\left\|\left(S^{\wedge}\right)^{1 / 2} F^{\wedge}\{f-g\}\right\|^{2}=\int_{0}^{\infty} u^{2 \omega}|f(u)-f(\theta u)|^{2} u d u,
\end{gathered}
$$

it is apparent that by taking $\theta$ sufficiently near 1 we can insure that

$$
\|f-g\|<\varepsilon / 2,\left\|\left(S^{\wedge}\right)^{1 / 2} F^{\wedge}\{f-g\}\right\|<\varepsilon / 2 .
$$

We next define $h(u)=g(u) \Delta_{1}(\lambda u)$. If $\lambda>0$ is so small that $\lambda+\theta<1$ then by Lemma 13a if $h^{*}=\psi^{-1} h \quad h^{*}(z)=0$ for $z>1$, and thus $F^{\wedge} h=$ $h$. Since 


$$
\begin{gathered}
\|g-h\|^{2}=\int_{0}^{\infty}|g(u)|^{2}|1-\Delta(\lambda u)|^{2} u d u, \\
\left\|\left(S^{\wedge}\right)^{1 / 2} F^{\wedge}\{g-h\}\right\|^{2}=\int_{0}^{\infty} u^{2 \omega}|g(u)|^{2}|1-\Delta(\lambda u)|^{2} u d u,
\end{gathered}
$$

it is evident that we can choose $\lambda>0$ so small that $\lambda+\theta<1$, and that

$$
\|g-h\|<\varepsilon / 2, \quad\left\|\left(S^{\wedge}\right)^{1 / 2} F^{\wedge}\{g-h\}\right\|<\varepsilon / 2 .
$$

Thus $h$ satisfiies (1). By Lemma 13a $h \in \boldsymbol{D}_{1}^{\wedge}$, and therefore by Theorem $12 \mathrm{~b}(2)$ holds as well.

Suppose that $F^{\wedge} f=0$. Then, if $f^{*}=\psi^{-1} f, f^{*}(z)=0$ for $0<z<1$. Choose $1<c_{1}<c_{2}<\infty$ so that if $g^{*}(z)=f^{*}(z)$ for $c_{1}<z<c_{2}$ and $g^{*}(z)=0$ otherwise then $\left\|f^{*}-g^{*}\right\|<\varepsilon / 2$. Let $g=\psi g^{*}$. Clearly $F^{\wedge} g=0$. We have

$$
\|f-g\|=\left\|f^{*}-g^{*}\right\|<\varepsilon / 2,
$$

while

$$
\left\|\left(S^{\wedge}\right)^{1 / 2} F^{\wedge}\{f-g\}\right\|=0 .
$$

Next let $h(u)=\Delta_{1}(\lambda u) g(u)$, where $\lambda>0$ is so small that $c_{1}-\lambda>1$, which implies using Lemma $13 \mathrm{~b}$, that $F^{\wedge} h=0$, and so small that $\|g-h\|<\varepsilon / 2$. Then $\|f-h\|<\varepsilon$ and $\left\|\left(S^{\wedge}\right)^{1 / 2} F^{\wedge}\{f-h\}\right\|=0$, so that (1) holds. By Lemmas 13a and $13 \mathrm{~b} h \in \boldsymbol{D}_{2}^{\wedge}$ and thus Theorem 12d can be applied to verify (2).

14. The asymptotic formula. Let $S_{F}^{\wedge}$ be constructed from $F^{\wedge}$ and $S^{\wedge}$ as in $\S 3$. Note that if $S^{\wedge}=\left\{f \mid f \in \boldsymbol{H}, F^{\wedge} f \in \boldsymbol{D}\left(S^{\wedge}\right)^{1 / 2}\right\}$ then $\boldsymbol{S}^{\wedge}$ is dense in $\boldsymbol{H}^{\wedge}$ so that $S_{F}^{\wedge}$ is a self-adjoint transformation on $\boldsymbol{H}$ itself. Let

$$
S_{F}^{\wedge}=\int_{0-}^{\infty} \lambda d \Psi^{\wedge}(\lambda)
$$

be the spectral resolution of $S_{F}^{\wedge}$ on $\boldsymbol{H}$, and let

$$
S_{n F}^{\wedge}=\int_{0-}^{\infty} \lambda d \Psi_{n}^{\wedge}(\lambda)
$$

be the spectral resolution of $S_{n, F}^{\wedge}=F_{n}^{\wedge} S_{n}^{\wedge} F_{n}^{\wedge}$. It follows from Theorems $9 \mathrm{~b}, 10 \mathrm{a}$ and 13c combined with Theorem 6a that

$$
\Psi_{n}^{\wedge}(\lambda) \rightarrow \Psi^{\wedge}(\lambda) \quad 0 \leqq \lambda<\infty
$$

for evey $\lambda \notin \sigma_{p}\left(S_{F}^{\wedge}\right)$.

Let us define 


$$
\begin{array}{ll}
R^{\wedge}=\left.S_{F}^{\wedge}\right|_{N^{\wedge},} & N^{\wedge}=F^{\wedge} H^{\wedge}, \\
R_{n}^{\wedge}=\left.S_{n, F}^{\wedge}\right|_{N_{n}^{\wedge},} & N_{n}^{\wedge}=F_{n}^{\wedge} H^{\wedge} .
\end{array}
$$

Since, as is easily seen, $R^{\wedge}>0, R_{n}^{\wedge}>0$, we have the spectral resolutions

$$
R^{\wedge}=\int_{0}^{\infty} \lambda d E^{\wedge}(\lambda) \quad \text { on } N^{\wedge}
$$

where

$$
E^{\wedge}(\lambda)=\Psi^{\wedge}(\lambda)-\Psi^{\wedge}(0) \quad 0 \leqq \lambda<\infty,
$$

and

$$
R_{n}^{\curlywedge}=\int_{0}^{\infty} \lambda d E_{n}^{\wedge}(\lambda)
$$

where

$$
E_{n}^{\wedge}(\lambda)=\Psi_{n}^{\wedge}(\lambda)-\Psi_{n}^{\wedge}(0) \quad 0 \leqq \lambda<\infty .
$$

Since $\Psi^{\wedge}(0)=I-F^{\wedge}, \Psi_{n}^{\wedge}(0)=I-F_{n}^{\wedge}$, it follows from (1) that

$$
E_{n}^{\wedge}(\lambda) \rightarrow E^{\wedge}(\lambda)
$$$$
0 \leqq \lambda<\infty
$$

for all $\lambda \notin \sigma_{p}(R)$.

LEMMA 14a. With the above definitions let $f_{n} \in N_{n}^{\wedge}$, and let $\left\|f_{n}\right\|=1, \quad\left(R_{n}^{\wedge} f_{n} \mid f_{n}\right) \leqq m<\infty$ for $n \in \mathfrak{p}$. We assert that if $f_{n} \rightarrow f$ as $n \rightarrow \infty$ in $\mathfrak{p}_{1}$ then $f \neq 0$.

Proof. If $f_{n} \in N_{n}^{\wedge}$ then

$$
f_{n}(u)=n^{-1} \sum_{k=1}^{n} R(k, n, u) a(k, n) \quad 0 \leqq u \leqq n \pi
$$

and $f_{n}(u)=0$ for $u>n \pi$. We have

$$
1=\left\|f_{n}\right\|^{2}=n^{-1} \sum_{k=0}^{n}|a(k, n)|^{2} .
$$

By Schwartz's inequality

$$
\left|f_{n}(u)\right|^{2} \leqq n^{-1} \sum_{k=0}^{n} R(k, n, u)^{2} .
$$

Since, see $\S 10$ for a similar estimate, if $0 \leqq k \leqq n$

$$
|R(k, n, u)| \leqq M\left(\frac{k+1}{n}\right)^{\alpha+(1 / 2)} u^{\alpha} \quad 0 \leqq u \leqq a_{2}<\infty,
$$


it follows that

$$
\left|f_{n}(u)\right| \leqq M u^{\alpha}
$$$$
0 \leqq u \leqq a_{2}
$$

Next

$$
\left|f_{n}^{\prime}(u)\right|^{2} \leqq n^{-1} \sum_{k=0}^{n} R^{\prime}(k, n, u)^{2} .
$$

Since, as is easily verified,

$$
\left|R^{\prime}(k, n, u)\right| \leqq M\left(\frac{k+1}{n}\right)^{\alpha+(1 / 2)} u^{\alpha-1} \quad 0<u \leqq a_{2}
$$

we have

$$
\left|f_{n}^{\prime}(u)\right| \leqq M u^{\alpha-1}
$$$$
0<u \leqq a_{2}
$$

It follows from (3) and (4) that the $\left\{f_{n}(u)\right\}_{1}^{\infty}$ are uniformly bounded and equicontinuous on any interval $0<a_{1} \leqq u \leqq a_{2}<\infty$. Therefore since $f_{n}(u) \rightarrow f(u)$ as $n$ in $\mathfrak{p}_{1}$ we have (if $f(u)$ is suitably redefined on a set of measure zero)

$$
\lim _{\mathfrak{p}_{1}} f_{n}(u)=f(u) \quad \text { uniformly for } a_{1} \leqq u \leqq a_{2} \text {. }
$$

Given any number $m_{1}>0$ we assert that there exists a number $a_{2}>0$ and an integer $N$ such that if $n \geqq N$

$$
s_{n}(u) \geqq m_{1} \quad a_{2} \leqq u \leqq n \pi .
$$

The inequality (6) is an immediate consequence of the relations

$$
\begin{aligned}
& s_{n}(u)=\left(2 n \sin \frac{u}{2 n}\right)^{2 \omega}\left[L\left(2 \sin ^{2} \frac{u}{2 n}\right) / L\left(n^{-2}\right)\right], \\
& s_{n}(u) \geqq M(\varepsilon)\left(2 n \sin \frac{u}{2 n}\right)^{2 \omega}\left[\left(2 n^{2} \sin ^{2} \frac{u}{2 n}\right)^{\varepsilon}+\left(2 n^{2} \sin ^{2} \frac{u}{2 n}\right)^{-\varepsilon}\right]^{-1} .
\end{aligned}
$$

See $\S 9$. We have

$$
\begin{gathered}
\int_{0}^{a_{2}} s_{n}(u)\left|f_{n}(u)\right|^{2} u d u+\int_{a_{2}}^{\infty} s_{n}(u)\left|f_{n}(u)\right|^{2} u d u=\left(R_{n}^{\wedge} f_{n} \mid f_{n}\right), \\
\int_{a_{2}}^{\infty} s_{n}(u)\left|f_{n}(u)\right|^{2} u d u \leqq m .
\end{gathered}
$$

By (6) if $n \geqq N$

$$
\int_{a_{2}}^{\infty} s_{n}(u)\left|f_{n}(u)\right|^{2} u d u \geqq m_{1} \int_{a_{2}}^{\infty}\left|f_{n}(u)\right|^{2} u d u .
$$

Therefore if $n \geqq N$ we have

$$
m_{1} \int_{a_{2}}^{\infty}\left|f_{n}(u)\right|^{2} u d u \leqq m
$$


and thus, since $\left\|f_{n}\right\|=1$,

$$
\int_{0}^{a_{2}}\left|f_{n}(u)\right|^{2} u d u \geqq 1-\frac{m}{m_{1}} .
$$

The relations (3), (5), and (7) imply that

$$
\int_{0}^{a_{2}}|f(u)|^{2} u d u \geqq 1-\frac{m}{m_{1}}>0
$$

and thus that $f \neq 0$ in $\boldsymbol{H}^{\wedge}$, as desired.

Applying Theorem $7 \mathrm{a}$ we now see that if $0<\mu_{1} \leqq \mu_{2} \leqq \mu_{3} \cdots$, $\lim _{k \rightarrow \infty} \mu_{k}=\infty$, are the eigen values of $S_{F}^{\wedge}$ then

$$
\lim _{n \rightarrow \infty} 2^{\omega} n^{2 \omega} L\left(n^{-2}\right)^{-1}\left[t(1)-\lambda_{n, k}\right]=\mu_{k} \quad k=1,2, \cdots .
$$

We have thus proved the following.

THEOREM 14b. Under the assumptions of $\S 8$ we have

$$
\lambda_{n, k}=t(1)-2^{-\omega} L\left(n^{-2}\right) n^{-2 \omega}\left[\mu_{k}+o(1)\right]
$$

as $n \rightarrow \infty$ for each fixed $k=1,2, \cdots$.

If we take $\omega=1, L(y)=\sigma$ then we obtain as a very special case of Theorem $14 \mathrm{~b}$ formula (7) of $\S 1$.

15. Maximum at an interior point. We will next take up the case where $t(x)$ has a unique absolute maximum at $x_{0},-1<x_{0}<1$. We assume that $t(x)$ is continuous for $-1 \leqq x \leqq 1$ and that

$$
t(x)<t\left(x_{0}\right) \quad-1 \leqq x \leqq 1, x \neq x_{0} .
$$

We further assume that

$$
t\left(x_{0}\right)-t(x)= \begin{cases}\sigma_{1}\left|x-x_{0}\right|^{\omega} L\left(x-x_{0}\right) & x \rightarrow x_{0}^{+} \\ \sigma_{2}\left|x-x_{0}\right|^{\omega} L\left(x-x_{0}\right) & x \rightarrow x_{0}^{-}\end{cases}
$$

where $\sigma_{1}>0, \sigma_{2}>0, \omega>0$ and $L(y)$ is a positive even function defined for $-2 \leqq y \leqq 2$ and continuous there except at $y=0$. At $y=0 L(y)$ is slowly oscillating.

In what follows we will again find it necessary to work with four Hilbert spaces.

$\boldsymbol{L}$ is, as before, the Hilbert space of complex valued functions $f(k)$ defined for $k=0,1,2, \cdots$, with inner product

$$
(f \mid g)_{L}=\sum_{k=0}^{\infty} f(k) g(k)^{*}
$$


Similarly $\boldsymbol{L}^{\wedge}$ is, as before, the space of Lebesgue measurable functions on $-1 \leqq x \leqq 1$ with inner product

$$
(f \mid g)_{\mathbf{L}^{\wedge}}=\int_{-1}^{1} f(x) g(x)^{*} w_{\alpha, \beta}(x) d x
$$

where $w_{a \beta}(x)$ is defined in $\S 1$.

$\boldsymbol{E}^{\wedge}$ and $\boldsymbol{E}$ are Hilbert spaces of Lebesgue measurable functions on $(-\infty, \infty)$ with inner products

$$
\begin{aligned}
(f \mid g)_{E^{\curlywedge}} & =\int_{-\infty}^{\infty} f(u) g(u)^{*} d u, \\
(f \mid g)_{E} & =\int_{-\infty}^{\infty} f(z) g(z)^{*} d z .
\end{aligned}
$$

We have the following maps between these spaces. There is, as before, a mapping $\phi$ from $\boldsymbol{L}$ to $\boldsymbol{L}^{\wedge}$ defined by

$$
\phi f \cdot(x)=\sum_{k=0}^{\infty} f(k) h_{k}^{-1 / 2} P_{k}^{(\alpha, \beta)}(x) .
$$

The series on the right is the limit of the partial sums in the metric of $\boldsymbol{L}^{\wedge}$. The inverse mapping is

$$
\phi^{-1} f \cdot(k)=\int_{-1}^{1} f(x) h_{k}^{-1 / 2} P_{k}^{(\alpha, \beta)}(x) w_{\alpha, \beta}(x) d x .
$$

These mappings are unitary.

There is a mapping $\psi$ from $\boldsymbol{E}$ to $\boldsymbol{E}^{\wedge}$ defined by

$$
\psi f \cdot(u)=\int_{-\infty}^{\infty} e^{2 \pi i u z} f(z) d z
$$

where the integral on the right is the limit of the partial integrals in the metric of $\boldsymbol{E}^{\wedge}$. The inverse mapping is

$$
\psi^{-1} f \cdot(z)=\int_{-\infty}^{\infty} e^{-2 \pi i u z} f(u) d u
$$

where etc. These mappings are also unitary.

Let $0<\xi_{0}<\pi$ be such that $\cos \xi_{0}=x_{0}$. We set

$$
\begin{aligned}
& \theta_{n}(u)= \\
& \quad\left[1-\cos \left(2 \pi u n^{-1}+\xi_{0}\right)\right]^{\alpha / 2}\left[1+\cos \left(2 \pi u n^{-1}+\xi_{0}\right)\right]^{\beta / 2} \sin ^{1 / 2}\left(2 \pi u n^{-1}+\xi_{0}\right) .
\end{aligned}
$$

For each $n=1,2, \cdots$ we define a mapping $\chi_{n}$ from $\boldsymbol{L}^{\wedge}$ to $\boldsymbol{E}^{\wedge}$ by setting

$$
\chi_{n} f \cdot(u)=\left\{\begin{array}{cr}
f\left(\cos \left[2 \pi u n^{-1}+\xi_{0}\right)\right] \theta_{n}(u)(2 \pi / n)^{1 / 2} & 0 \leqq 2 \pi u n^{-1}+\xi_{0} \leqq \pi \\
0 & \text { otherwise . }
\end{array}\right.
$$


Clearly $\chi_{n}$ is an isometric map of $\boldsymbol{L}^{\wedge}$ into $\boldsymbol{E}^{\wedge}$. We define a mapping: $\chi_{n}^{*}$ from $\boldsymbol{E}^{\wedge}$ to $\boldsymbol{L}^{\wedge}$ by

$$
\chi_{n}^{*} f \cdot(x)=f\left(\frac{n}{2 \pi}\left[-\xi_{0}+\arccos x\right]\right)\left(1-x^{2}\right)^{-1 / 4} w_{\alpha, \beta}(x)^{-1 / 2}(n / 2 \pi)^{1 / 2} .
$$

$\chi_{n}^{*}$ is a partially isometric mapping of $\boldsymbol{E}^{\wedge}$ onto $\boldsymbol{L}^{\wedge}$. $\chi_{n}^{*}$ is isometric on $\chi_{n} \boldsymbol{L}^{\wedge}$ and zero on $\left(\chi_{n} \boldsymbol{L}^{\wedge}\right)^{\perp}$, the orthogonal complement of $\chi_{n} \boldsymbol{L}^{\wedge}$ in $\boldsymbol{E}^{\wedge}$. Moreover $\chi_{n}^{*} \chi_{n}=I$ and $\chi_{n} \chi_{n}^{*}=I$ on $\chi_{n} L^{\wedge}$ and $\chi_{n} \chi_{n}^{*}=0$ on the orthogonal complement of $\chi_{n} \boldsymbol{L}^{\wedge}$.

We now introduce various operators on these Hilbert spaces.

a. $E_{n}$ is defined on $\boldsymbol{L}$ by

$$
E_{n} f \cdot(k)=\left\{\begin{array}{cc}
f(k) & 0 \leqq k \leqq n \\
0 & \text { otherwise } .
\end{array}\right.
$$

$E_{n}$ induces the following additional operators:

$$
\begin{aligned}
& E_{n}^{\wedge} \text { on } \boldsymbol{L}^{\wedge} \text { defined by } E_{n}^{\wedge}=\phi E_{n} \phi^{-1} ; \\
& F_{n}^{\wedge} \text { on } \boldsymbol{E}^{\wedge} \text { defined by } F_{n}^{\wedge}=\chi_{n} E_{n}^{\wedge} \chi_{n}^{*} .
\end{aligned}
$$

b. $\quad T^{\wedge}$ is defined on $\boldsymbol{L}^{\wedge}$ by

$$
T^{\wedge} f \cdot(x)=\left[t\left(x_{0}\right)-t(x)\right] f(x) .
$$

Starting from $T^{\wedge}$ we obtain the following related operators:

$$
\begin{aligned}
& T \text { on } \boldsymbol{L} \text { defined by } T=\phi^{-1} T^{\wedge} \phi ; \\
& T_{n}^{\wedge} \text { on } \boldsymbol{E}^{\wedge} \text { defined by } T_{n}=\chi_{n} T^{\wedge} \chi_{n}^{*}: \\
& S_{n}^{\wedge} \text { on } \boldsymbol{E}^{\wedge} \text { defined by } S_{n}^{\wedge}=\left[n^{\omega} / L\left(n^{-1}\right)\right] T_{n}^{\wedge} .
\end{aligned}
$$

c. $S^{\wedge}$ is defined on $\boldsymbol{E}^{\wedge}$ by

$$
S^{\wedge} f(u)=f(u) s(u)
$$

where

$$
s(u)= \begin{cases}\sigma_{1}\left(-2 \pi\left[\sin \xi_{0}\right] u\right)^{\omega} & u \leqq 0 \\ \sigma_{2}\left(2 \pi\left[\sin \xi_{0}\right] u\right)^{\omega} & u>0 .\end{cases}
$$

d. $F$ is defined on $\boldsymbol{E}$ by

$$
F f \cdot(z)=\left\{\begin{array}{cl}
f(z) & |z| \leqq 1 \\
0 & |z|>1 .
\end{array}\right.
$$

We introduce

$$
F^{\wedge} \text { on } E^{\wedge} \text { defined by } F^{\wedge}=\psi F \psi^{-1} \text {. }
$$

If $\lambda_{n, 1} \geqq \lambda_{n, 2} \geqq \cdots \geqq \lambda_{n n+1}$ are the eigen values of $C_{n}$, see $\S 1$, then 


$$
t\left(x_{0}\right)-\lambda_{n, 1} \leqq t\left(x_{0}\right)-\lambda_{n, 2} \leqq \cdots \leqq t\left(x_{0}\right)-\lambda_{n, n+1}
$$

are the eigen values of the following operators:

$$
\begin{gathered}
E_{n} T E_{n} \mid E_{n} \boldsymbol{L} ; \\
E_{n}^{\wedge} T^{\wedge} E_{n}^{\wedge} \mid E_{n}^{\wedge} \boldsymbol{L}^{\wedge} ; \\
F_{n}^{\wedge} T_{n}^{\wedge} F_{n}^{\wedge} \mid F_{n}^{\wedge} E^{\wedge} .
\end{gathered}
$$

The eigen values of

$$
F_{n}^{\wedge} S_{n}^{\wedge} F_{n}^{\wedge} \mid F_{n}^{\wedge} E^{\wedge}
$$

are, in increasing order, $\left\{\left[t\left(x_{0}\right)-\lambda_{n, k}\right] n^{\omega} L\left(n^{-1}\right)^{-1}\right\}_{k=1}^{n+1}$. Our program in what follows is like that carried out in sections 8-14, in that we will show that $F_{n}^{\wedge} S_{n}^{\wedge} F_{n}^{\wedge}$ "converges" to $S_{F}^{\wedge}$ as $n \rightarrow \infty$ and thus that if

$$
0<\mu_{1} \leqq \mu_{2} \leqq \cdots, \lim _{k \rightarrow \infty} \mu_{k}=\infty,
$$

are the eigen values of

$$
\boldsymbol{S}_{F}^{\wedge} \mid F^{\wedge} \boldsymbol{E}^{\wedge}
$$

then

$$
\lim _{n \rightarrow \infty}\left(t\left(x_{0}\right)-\lambda_{n, k}\right) n^{\omega} L\left(n^{-1}\right)^{-1}=\mu_{k} \quad k=1,2, \cdots,
$$

or equivalently

$$
\lambda_{n, k}=t\left(x_{0}\right)-n^{-\omega} L\left(n^{-1}\right)\left[\mu_{k}+o(1)\right]
$$

as $n \rightarrow \infty$.

Because the material of $\S \S 15-19$ is in large part like the material of $\S \S 8-14$ we will only give in detail those arguments which differ from those given there. These occur primarily in $\S 16$ and $\S 17$. In the later sections we will simply list the various results since the details can be easily supplied.

16. Convergence of $\left(S_{n}^{\wedge}\right)^{1 / 2}$ to $\left(S^{\wedge}\right)^{1 / 2}$ (interior maximum). We suppose throughout that $t(x)$ satisfies the assumptions of $\S 15$. Let $0<\xi_{0}<\pi$ be such that $\cos \xi_{0}=x_{0}$.

It follows from $\S 15$ that $T_{n}^{\wedge} f(u)=t_{n}(u) f(u)$ where $t_{n}(u)=$ $t\left(\cos \xi_{0}\right)-t\left[\cos \left(2 \pi n^{-1} u+\xi_{0}\right)\right]$ for $0 \leqq 2 \pi n^{-1} u+\xi_{0} \leqq \pi$ and is zero otherwise. Consequently $S_{n}^{\wedge} f(u)=s_{n}(u) f(u)$ where $s_{n}(u)=t_{n}(u) n^{\omega} L\left(n^{-1}\right)^{-1}$. Let $s(u)$ be defined as in $\S 15$. 


$$
\lim _{n \rightarrow \infty} s_{n}(u)=s(u)
$$$$
-\infty<u<\infty,
$$

and for any $\varepsilon>0$ there is a constant $M(\varepsilon)$ independent of $u$, $-\infty<u<\infty$, and $n=1,2, \cdots$ such that

$$
s_{n}(u) \leqq M(\varepsilon)\left[|u|^{\varepsilon}+|u|^{-\varepsilon}\right]|u|^{\omega} .
$$

Proof. It follows from the assumptions of $\S 15$ that if $\lambda(u)=$ $\cos \left(2 \pi u+\xi_{0}\right)-\cos \xi_{0}$ then

$$
t\left(\cos \xi_{0}\right)-t \cos \left(2 \pi u+\xi_{0}\right) \sim \begin{cases}\sigma_{2}|\lambda(u)|^{\omega} L(\lambda(u)) & u \rightarrow 0+ \\ \sigma_{1}|\lambda(u)|^{\omega} L(\lambda(u)) & u \rightarrow 0-.\end{cases}
$$

Since $\lambda(u)-2 \pi u \sin \xi_{0}$ as $u \rightarrow 0$ we find using (4) of $\S 9$ that

(3) $\left[t\left(\cos \xi_{0}\right)-t \cos \left(2 \pi u+\xi_{0}\right)\right]-\left\{\begin{array}{ll}\sigma_{2}\left(2 \pi \sin \xi_{0}\right)^{\omega} u^{\omega} L(u) & \text { as } u \rightarrow 0+ \\ \sigma_{1}\left(2 \pi \sin \xi_{0}\right)^{\omega}(-u)^{\omega} L(n) & \text { as } u \rightarrow 0-\end{array}\right.$.

Thus for $u$ fixed, $u \neq 0$, we see that as $n \rightarrow \infty$

$$
s_{n}(u)- \begin{cases}\sigma_{2}\left(2 \pi \sin \xi_{0}\right)^{\omega} u^{\omega} L\left(u n^{-1}\right) / L\left(n^{-1}\right) & u>0 \\ \sigma_{1}\left(2 \pi \sin \xi_{0}\right)^{\omega}(-u)^{\omega} L\left(u n^{-1}\right) / L\left(n^{-1}\right) & u<0 .\end{cases}
$$

A second application of (4) of $\S 9$ yields (1). It follows from (3) that if $b$ is a sufficiently large positive constant then

$$
t\left(\cos \xi_{0}\right)-t\left[\cos \left(2 \pi u+\xi_{0}\right)\right] \leqq b|u|^{\omega} L(u),
$$

and thus

$$
s_{n}(u) \leqq b|u|^{\omega} L\left(u n^{-1}\right) / L\left(n^{-1}\right) .
$$

Using (5) of $\S 9$ we obtain our desired result.

THEOREM 16b. $\left(S^{\wedge}\right)^{1 / 2}$ is the closure of the strong limit of $\left(S_{n}^{\wedge}\right)^{1 / 2}$ as $n \rightarrow \infty$.

Proof. Let $f \in D\left[\left(S^{\wedge}\right)^{1 / 2}\right]$ and $\varepsilon>0$ be given. If $\delta>0$ is sufficiently small then it is evident that if $f_{\delta}(u)=e^{-\delta u^{2}} f(u)$ then

$$
\left\|f-f_{\delta}\right\|_{E^{\curvearrowleft}} \leqq \varepsilon, \quad\left\|\left(S^{\wedge}\right)^{1 / 2}\left(f-f_{\delta}\right)\right\|_{E^{\wedge}} \leqq \varepsilon .
$$

Moreover it is evident from (1) and (2) that

$$
\left(S_{n}^{\wedge}\right)^{1 / 2} f_{\delta} \rightarrow\left(S^{\wedge}\right)^{1 / 2} f_{\delta} \quad \text { in } \boldsymbol{E}^{\wedge} \text { as } n \rightarrow \infty .
$$

17. Convergence of $F_{n}^{\wedge}$ (interior maximum).

TheoRem 17a. If $F_{n}^{\wedge}$ and $F^{\wedge}$ are defined as in $\S 15$ then $F_{n}^{\wedge}$ 
converges strongly to $F^{\wedge}$ as $n \rightarrow \infty$.

Proof. Let us write

$$
\begin{aligned}
& R(k, n, u)=h_{k}^{-1 / 2} P_{k}^{(\alpha, \beta)}\left(\cos 2 \pi u n^{-1}+\xi_{0}\right) \theta_{n}(u) \sqrt{2 \pi} . \\
& Q(k, n, u)=\cos \left(2 \pi(k+\eta) n^{-1} u+k \xi_{0}+\zeta\right),
\end{aligned}
$$

where $\eta=(\alpha+\beta+1) / 2, \zeta=\xi_{0}(\alpha+\beta+1) / 2-(\alpha+(1 / 2)) \pi / 2$. It follows from (6) of the Appendix that

$$
R(k, n, u)-2 Q(k, n, u) \rightarrow 0
$$

as $k \rightarrow \infty$

uniformly in $n$ and $u$, if for some fixed $\varepsilon>0$

$$
\varepsilon \leqq 2 \pi u n^{-1}+\xi_{0} \leqq \pi-\varepsilon .
$$

Starting from the definition of $F_{n}^{\wedge}$ we find that

$$
F_{n}^{\wedge} f \cdot(u)=\frac{1}{n} \sum_{k=0}^{n} R(k, n, u) a(k, n)
$$

where

$$
a(k, n)=\int_{I_{n}} f(v) R(k, n, v) d v .
$$

Here $I_{n}=\left\{v \mid 0 \leqq 2 \pi n^{-1} v+\xi_{0} \leqq \pi\right\}$.

Let us now assume that $f(u)$ is continuous for $-\infty<u<\infty$ and vanishes except for $|u| \leqq a$. We first show that under this assumption $F_{n}^{\wedge} f \cdot(u) \rightarrow F^{\wedge} f \cdot(u)$ uniformly in any set $|u| \leqq b<\infty$. It follows from (1) that there exists a constant $M$ such that if $n$ is sufficiently large.

$$
|R(k, n, u)| \leqq M
$$

for $|u| \leqq a$, and $k=0,1, \cdots$. Let us set

$$
\begin{aligned}
& \sum_{1}(\delta, n, u)=\frac{1}{n} \sum_{0 \leqq k<n \delta} R(k, n, u) a(k, n), \\
& \sum_{2}(\delta, n, u)=\frac{1}{n} \sum_{n \delta \leqq k \leqq n} R(k, n, u) a(k, n) .
\end{aligned}
$$

Using (4) and the corresponding inequality for $|u| \leqq b$ we find that for all large $n$

$$
\sum_{1}(\delta, n, u) \leqq M \delta \quad \text { if }|u| \leqq b .
$$

Let $g=\psi^{-1} f$ so that

$$
g(z)=\int_{-\infty}^{\infty} f(u) e^{-2 \pi i u z} d u
$$


Using (1), but writing the cosine in complex form, we find that

$$
\lim _{k \rightarrow \infty}\left\{a(n, k)-e^{-i\left(k \xi_{0}+\zeta\right)} g\left(\frac{k+\eta}{n}\right)-e^{i\left(k \varepsilon_{0}+\zeta\right)} g\left(-\frac{k+\eta}{n}\right)\right\}=0 .
$$

Using (1) again we see that as $n \rightarrow \infty$

$$
\left|\sum_{2}(\delta, n, u)-\Sigma_{I}-\sum_{I I}-\sum_{I I I}-\Sigma_{I V}\right| \rightarrow 0
$$

uniformly for $|u| \leqq b$ where

$$
\begin{aligned}
\sum_{I} & =n^{-1} \sum_{\delta n \leqq k \leqq n} e^{2 \pi i(k+\eta) u n-1} g\left(\frac{k+\eta}{n}\right), \\
\sum_{I I} & =n^{-1} \sum_{\delta n \leqq k \leqq n} e^{2 i\left(k \xi_{0}+\zeta\right)} e^{2 \pi i(k+\eta) u n^{-1}} g\left(-\frac{k+\eta}{n}\right), \\
\sum_{I I I} & =n^{-1} \sum_{\delta n \leqq k \leqq n} e^{-2 \pi i(k+\eta) u n-1} g\left(-\frac{k+\eta}{n}\right), \\
\sum_{I V} & =n^{-1} \sum_{\delta n \leqq k \leqq n} e^{-2 i\left(k \xi_{0}+\zeta\right)} e^{-2 \pi i(k+\eta) u n-1} g\left(\frac{k+\eta}{n}\right) .
\end{aligned}
$$

$\sum_{I}$ and $\sum_{I I I}$ are Riemann sums so that

$$
\begin{aligned}
\lim _{n \rightarrow \infty} \sum_{I} & =\int_{\delta}^{1} e^{2 \pi i u z} g(z) d z, \\
\lim _{n \rightarrow \infty} \sum_{I I I} & =\int_{-1}^{-\delta} e^{2 \pi i u z} g(z) d z,
\end{aligned}
$$

for $|u| \leqq b$. Since we can easily prove that the functions $\sum_{I}(\delta, n, u)$ are for $n$ sufficiently large equicontinuous for $|u| \leqq b$ it follows that (7) holds uniformly for $|u| \leqq b$. Similar remarks apply to $\sum_{I I I}$. If we sum $\sum_{I I}$ by parts, the summation being applied to $e^{-2 i k \xi_{0}}$, it is easily seen that $\lim _{n \rightarrow \infty} \sum_{I I}=0$ uniformly for $|u| \leqq b$ and similarly for $\sum_{I V}$. Given $\varepsilon>0$ let us choose $\delta$ so small that

$$
\left|\sum_{1}(\delta, n, u)\right|<\varepsilon / 2, \quad\left|\int_{-\delta}^{\delta} g(z) e^{2 \pi i u z} d z\right|<\varepsilon / 2,
$$

for $|u| \leqq b$. It then follows on collecting our estimates that $\left|F_{n}^{\wedge} f \cdot(u)-F^{\wedge} f \cdot(u)\right|<\varepsilon$ for $|u| \leqq b$, for all sufficiently large $n$. The demonstration can be completed as in $\S 10$.

18. Convergence of $\left(S_{n}^{\wedge}\right)^{1 / 2} F_{n}^{\wedge}$ to $\left(S^{\wedge}\right)^{1 / 2} F^{\wedge}$ (interior maximum). The considerations here are parallel to those of $\S 11, \S 12$, and $\S 13$ but somewhat simpler.

Let $\boldsymbol{D}$ be the set of functions $h(z)$ in $\boldsymbol{E}$ which are infinitely differentiable and have compact support, and let $\boldsymbol{D}^{\wedge}=\psi \boldsymbol{D}$. Let $\boldsymbol{D}_{\boldsymbol{1}}$ be the subset of $\boldsymbol{D}$ consisting of those functions which have support in 
$|z| \leqq c_{1}$ for some $c_{1}<1$, and let $\boldsymbol{D}_{1}^{\wedge}=\psi \boldsymbol{D}_{1}$. Let $\boldsymbol{D}_{2}$ be the subset of $D$ consisting of those functions which have support in $|z| \geqq c_{2}$ for some $c_{2}>1$, and let $\boldsymbol{D}_{2}^{\wedge}=\psi \boldsymbol{D}_{2}$.

THEOREM 18a. If $f \in \boldsymbol{D}_{1}^{\wedge}$ or $\boldsymbol{D}_{2}$ and if, as in $\S 17$,

$$
a(k, n)=\int_{I_{n}} f(\zeta) R(k, n, \zeta) d \zeta,
$$

then for $\nu$ fixed, $\nu=0, \pm 1, \pm 2, \cdots$, we have

$$
a(n, n+\nu)=0\left(n^{-r}\right)
$$

for every $r$.

Proof. We will carry out only the first steps of the demonstration since it will be evident in a moment that the arguments used in $\S 11$ apply almost without change.

We recall that $I_{n}=\left\{\zeta \mid-\gamma_{1} n \leqq \zeta \leqq \gamma_{2} n\right\}$ where $\gamma_{2}=\left(\pi-\xi_{0}\right) / 2 \pi$, $\gamma_{1}=\xi_{0} / 2 \pi$. Choose $\delta_{1}, 0<\delta_{1}<\gamma_{1}$ and $\delta_{2}, 0<\delta_{2}<\gamma_{2}$. Then

$$
a(k, n)=a_{1}(k, n)+a_{2}(k, n)+a_{3}(k, n)
$$

where

$$
\begin{aligned}
& a_{1}(k, n)=\int_{-\delta_{1} n}^{\delta_{2} n} f(\zeta) R(k, n, \zeta) d \zeta, \\
& a_{2}(k, n)=\int_{\delta_{2} n}^{\gamma_{2} n} f(\zeta) R(k, n, \zeta) d \zeta, \\
& a_{3}(k, n)=\int_{-\gamma_{1} n}^{-\delta_{1} n} f(\zeta) R(k, n, \zeta) d \zeta .
\end{aligned}
$$

Since $f(\zeta) \in \boldsymbol{D}^{\wedge}$ we have

$$
f(\zeta)=\int_{-\infty}^{\infty} g(z) e^{2 \pi i z \zeta} d z
$$

where $g=\psi^{-1} f$ is infinitely differentiable with compact support. Repeated integration by parts shows that

$$
f(\zeta)=0\left(|\zeta|^{-r}\right) \quad \zeta \rightarrow \pm \infty
$$

for every $r$. Using

$$
\int_{I_{n}} R(k, n, \zeta)^{2} d \zeta=n
$$

and Schwartz's inequality we see that

$$
\left|a_{2}(k, n)\right|^{2} \leqq n \int_{\delta_{2} n}^{\gamma_{2} n}|f(\zeta)|^{2} d \zeta
$$


and using (1) that $a_{2}(k, n)=0\left(n^{-r}\right)$ as $n \rightarrow \infty$ uniformly in $k$. Similar considerations apply to $a_{3}(k, n)$. If we set

$$
\begin{aligned}
& a_{1}^{+}(k, n)=\int_{-\delta_{1} n}^{\delta_{2} n} h_{k}^{-1 / 2} Q_{k}^{+}\left[\cos \left(2 \pi \zeta n^{-1}+\xi_{0}\right)\right] \Theta_{n}(\zeta) f(\zeta) d \zeta, \\
& a_{1}^{-}(k, n)=\int_{-\delta_{1} n}^{\delta_{2} n} h_{k}^{-1 / 2} Q_{k}^{-}\left[\cos \left(2 \pi \zeta n^{-1}+\xi_{0}\right)\right] \Theta_{n}(\zeta) f(\zeta) d \zeta,
\end{aligned}
$$

where

$$
\begin{array}{r}
\Theta_{n}(\zeta)=\left[1-\cos \left(2 \pi \zeta n^{-1}+\xi_{0}\right)\right]^{-\alpha / 2}\left[1+\cos \left(2 \pi \zeta n^{-1}+\xi_{0}\right)\right]^{-\beta / 2} \\
\cdot \sin ^{1 / 2}\left(2 \pi \zeta n^{-1}+\xi_{0}\right) \sqrt{2 \pi} .
\end{array}
$$

then

$$
-\pi i a_{1}(k, n)=a_{1}^{-}(k, n)-a_{1}^{+}(k, n) .
$$

Let us apply Cauchy's theorem to each of the curves below.

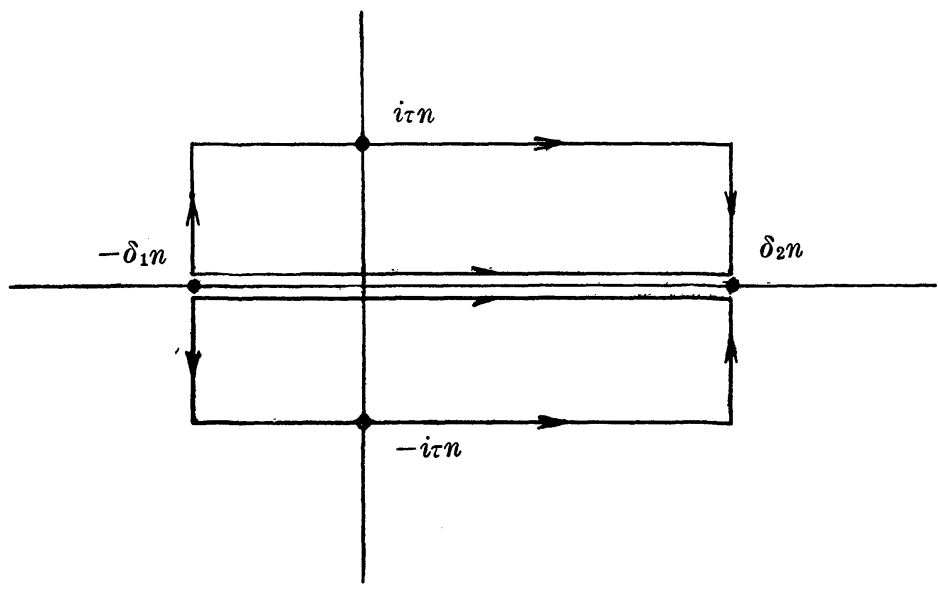

We find that

$$
a_{1}^{ \pm}(k, n)=I_{1}^{ \pm}+I_{2}^{ \pm}+I_{3}^{ \pm}
$$

where

$$
\begin{array}{rlrl}
I_{1}^{-} & =\int_{-\delta_{1} n}^{-\delta_{1} n+i \tau n}, & I_{2}^{-}=\int_{-\delta_{1} n+i \tau n}^{\delta_{2} n+i \tau n}, & I_{3}^{-}=\int_{\delta_{2} n+i \tau n}^{\delta_{2} n}, \\
I_{1}^{+}=\int_{-\delta_{1} n}^{-\delta_{1} n-i \tau n}, & I_{2}^{+}=\int_{-\delta_{1} n-i \tau n}^{\delta_{2} n-i \tau n}, & I_{3}^{+}=\int_{\delta_{2} n-i \tau n}^{\delta_{2} n} .
\end{array}
$$

In all cases the integrand is

$$
f(\zeta) h_{k}^{-1 / 2} Q_{k}\left[\cos \left(2 \pi \zeta n^{-1}+\xi_{0}\right)\right] \Theta_{n}(\zeta) d \zeta .
$$

It is sufficient to verify that each of these six integrals is $0\left(n^{-r}\right)$ ?as 
$n \rightarrow \infty$. Since the methods of $\S 11$ now apply almost without change the remainder of the proof for the case $f \in \boldsymbol{D}_{1}^{\wedge}$ is omitted, as well as the proof for the case $f \in \widehat{D_{2}}$.

Lemma 18b. Let $g \in \widehat{\boldsymbol{D}_{1}^{\wedge}}$ or $\widehat{\boldsymbol{D}_{2}}$. Then for any nonnegative integer $N$ and some finite constant $A_{N}$ we have

$$
\lim _{n \rightarrow \infty} \int_{I_{n}} u^{2 N}\left|F_{n}^{\wedge} g \cdot(u)\right|^{2} d u \leqq A_{N} \int_{-\infty}^{\infty} u^{2 N}|g(u)|^{2} d u .
$$

We will only sketch the proof of this result. Let

$$
\rho_{n}(u)=\left[\cos \left(2 \pi u n^{-1}+\xi_{0}\right)-\cos \xi_{0}\right]^{N} .
$$

Using the recursion formula and Theorem 18a it is easy to see that if $g_{n}(u)=\rho_{n}(u) g(u)$

$$
\rho_{n}(u) F_{n}^{\wedge} g \cdot(u)=F_{n}^{\wedge} g_{n} \cdot(u)+0\left(n^{-r}\right)
$$

for $u \in I_{n}$. It follows that

$$
\begin{aligned}
\varlimsup_{n \rightarrow \infty} \int_{I_{n}} \rho_{n}(u)^{2}\left|F_{n}^{\wedge} g \cdot(u)\right|^{2} d u & \leqq \varlimsup_{n \rightarrow \infty} \int_{I_{n}}\left|F_{n}^{\wedge} g_{n} \cdot(u)\right|^{2} d u, \\
& \leqq \varlimsup_{n \rightarrow \infty} \int_{-\infty}^{\infty}\left|g_{n}(u)\right|^{2} d u,
\end{aligned}
$$

since $F_{n}^{\wedge}$ is a projection; that is

$$
\varlimsup_{n \rightarrow \infty} \int_{I_{n}} \rho_{n}(u)^{2}\left|F_{n}^{\wedge} g \cdot(u)\right|^{2} d u \leqq \varlimsup_{n \rightarrow \infty} \int_{-\infty}^{\infty} \rho_{n}(u)^{2}|g(u)|^{2} d u .
$$

We have

$$
\cos \left(2 \pi u n^{-1}+\xi_{0}\right)-\cos \xi_{0}=-2 \sin \left(\pi u n^{-1}\right) \sin \left(\pi u n^{-1}+\xi_{0}\right) .
$$

Since $0 \leqq 2 \pi u n^{-1}+\xi_{0} \leqq \pi$ if $u \in I_{n}$ we have

$$
0<\xi_{0} / 2 \leqq \pi u n^{-1}+\xi_{0} \leqq\left(\pi+\xi_{0}\right) / 2 \quad \text { for } u \in I_{n} \text {. }
$$

It follows that there exist finite positive constants $A_{1}$ and $A_{2}$ such that

$$
\begin{array}{rlr}
\left|\left(\cos 2 \pi u n^{-1}+\xi_{0}\right)-\cos \xi_{0}\right| & \leqq A_{1}|u| & -\infty<u<\infty, \\
& \geqq A_{2}|u| & u \in I_{n},
\end{array}
$$

etc.

THEOREM 18c. Let $f \in \widehat{\boldsymbol{D}_{1}^{\wedge}}$ or $\boldsymbol{D}_{2}^{\wedge}$. Then

$$
\lim _{n \rightarrow \infty}\left\|\left(S^{\wedge}\right)^{1 / 2} F^{\wedge} f-\left(S_{n}^{\wedge}\right)^{1 / 2} F_{n}^{\wedge} f\right\|=0 .
$$


Note that if $f \in \boldsymbol{D}_{2}^{\wedge}, F^{\wedge} f=0$.

THEOREM 18d. $\left(S^{\wedge}\right)^{1 / 2} F^{\wedge}$ is the closure of the strong limit of $\left(S_{n}^{\wedge}\right)^{1 / 2} F_{n}^{\wedge}$ as $n \rightarrow \infty$.

Note that the demonstration of Theorem $18 \mathrm{~d}$ is simpler than that of its analogue, Theorem 13c, in that, because convolution is possible in $E$, the analogues of Lemmas $13 \mathrm{a}$ and $13 \mathrm{~b}$ are completely trivial.

19. The asymptotic formula (interior maximum). In this section we will complete the theory for the case of an interior maximum giving some details. Let $S_{F}^{\wedge}$ be constructed from $F^{\wedge}$ and $S^{\wedge}$ as in $\S 3$. Note that if $S^{\wedge}=\left\{f \mid f \in \boldsymbol{E}^{\wedge}, F^{\wedge} f \in \boldsymbol{D}\left(S^{\wedge}\right)^{1 / 2}\right\}$ then $\boldsymbol{S}^{\wedge}$ is dense in $\boldsymbol{E}^{\wedge}$ so that $S_{F}^{\wedge}$ is a self-adjoint transformation on $\boldsymbol{E}^{\wedge}$ itself. Let

$$
S_{F}^{\wedge}=\int_{0-}^{\infty} \lambda d \Psi^{\wedge}(\lambda)
$$

be the spectral resolution of $S_{F}^{\wedge}$ on $\boldsymbol{E}^{\wedge}$, and let

$$
S_{n, F^{\prime}}^{\wedge}=\int_{0-}^{\infty} \lambda d \Psi_{n}^{\wedge}(\lambda)
$$

be the spectral resolution of $S_{n F}^{\wedge}=F_{n}^{\wedge} S_{n}^{\wedge} F_{n}^{\wedge}$. It follows from Theorems 16b, 17a, and 18b, combined with Theorem 6a that

$$
\Psi_{n}^{\wedge}(\lambda) \rightarrow \Psi^{\wedge}(\lambda)
$$$$
0 \leqq \lambda<\infty
$$

for every $\lambda \notin \sigma_{p}\left(S_{F}^{\wedge}\right)$.

Let us define

$$
\begin{array}{ll}
R^{\wedge}=\left.S_{F}^{\wedge}\right|_{N^{\wedge}} & N^{\wedge}=F^{\wedge} E^{\wedge}, \\
R_{n}^{\wedge}=\left.S_{n, F}^{\wedge}\right|_{N_{n}^{\wedge}} & N_{n}^{\wedge}=F_{n}^{\wedge} E^{\wedge} .
\end{array}
$$

Since, as is easily seen, $R^{\wedge}>0, R_{n}^{\wedge}>0$, we have the spectral resolutions

$$
R^{\wedge}=\int_{0}^{\infty} \lambda d E^{\wedge}(\lambda) \text { on } N^{\wedge}, R_{n}^{\wedge}=\int_{0}^{\infty} \lambda d E_{n}^{\wedge}(\lambda) \text { on } N_{n}^{\wedge}
$$

where

$$
\begin{array}{ll}
E^{\wedge}(\lambda)=\Psi^{\wedge}(\lambda)-\Psi^{\wedge}(0) & 0 \leqq \lambda<\infty, \\
E_{n}^{\wedge}(\lambda)=\Psi_{n}^{\wedge}(\lambda)-\Psi_{n}^{\wedge}(0) & 0 \leqq \lambda<\infty .
\end{array}
$$

Since $\Psi^{\wedge}(0)=I-F^{\wedge}, \Psi_{n}^{\wedge}(0)=I-F_{n}^{\wedge}$ it follows from (1) that

$$
E_{n}^{\wedge}(\lambda) \rightarrow E^{\wedge}(\lambda)
$$$$
0 \leqq \lambda<\infty
$$ 
for all $\lambda \notin \sigma_{p}\left(R^{\wedge}\right)$.

Lemma 19a. With the above definitions let $f_{n} \in N_{n}^{\wedge}$, and let $\left\|f_{n}\right\|=1,\left(R_{n}^{\wedge} f_{n} \mid f_{n}\right) \leqq m$ for $n \in \mathfrak{p}$. We assert that if $f_{n} \rightarrow f$ as $n \rightarrow \infty$ in $\mathfrak{p}_{1}$ then $f \neq 0$.

Proof. If $f_{n} \in N_{n}^{\wedge}$ then

$$
f_{n}(u)=\frac{1}{n} \sum_{k=0}^{n} R(k, n, u) a(k, n)
$$

and $f_{n}(u)=0$ if $u \notin I_{n}$. Here $R(k, n, u)$ is defined as in $\S 17$, and

$$
\alpha(k, n)=\int_{I_{n}} R(k, n, \zeta) f_{n}(\zeta) d \zeta
$$

we have

$$
1=\left\|f_{n}\right\|^{2}=\frac{1}{n} \sum_{k=0}^{n}|a(n, k)|^{2},
$$

and therefore by Schwarz's inequality

$$
\left|f_{n}(u)\right|^{2} \leqq \frac{1}{n} \sum_{k=0}^{n} R(k, n, u)^{2} .
$$

(1) of $\S 17$ if $|u| \leqq \alpha<\infty$ then there exists a constant $M$ such lat $|R(k, n, u)| \leqq M$ for $k=0,1, \cdots$ provided $n$ is sufficiently large. follows that for all large $n$

3)

$$
\left|f_{n}(u)\right| \leqq M \quad|u| \leqq a .
$$

ext

$$
\left|f_{n}^{\prime}(u)\right|^{2} \leqq \frac{1}{n} \sum_{k=0}^{n} R^{\prime}(k, n, u)^{2}
$$

le assert that if $|u| \leqq a$ then for all sufficiently large $n$ and a suitable onstant $M,\left|R^{\prime}(k, n, u)\right| \leqq M$ for $k=0,1, \cdots, n$. This inequality an be reduced by means of the formula

$$
2 \frac{d}{d x} P_{n}^{(\alpha, \beta)}(x)=(n+\alpha+\beta+1) P_{n-1}^{(\alpha+1, \beta+1)}(x),
$$

o the one given above. See $[1 ; p .170]$. We may therefore apply chwarz's inequality again to obtain for all sufficiently large $n$

$$
\left|f_{n}^{\prime}(u)\right| \leqq M \quad|u| \leqq a .
$$

t follows from (3) and (4) that the $\left\{f_{n}(u)\right\}_{1}^{\infty}$ are uniformly bounded nd equicontinuous on any interval $|u| \leqq \alpha<\infty$. Therefore if $f_{n} \rightarrow f$ 
as $n \rightarrow \infty$ in $\mathfrak{p}_{1}$ we have

$$
\lim _{\mathfrak{p}_{1}} f_{n}(u)=f(u)
$$$$
|u| \leqq a
$$

uniformly, provided $f(u)$ is suitably redefined on a set of measure zero.

Given $m_{1}>0$ it is easy to see that there exists a number $a>0$ and an integer $N$ such that if $n \geqq N$

$$
s_{n}(u) \geqq m_{1} \quad\left\{u \in I_{n}|| u \mid \geqq a\right\} .
$$

The remainder of the proof follows the lines $\S 14$ so closely it is omitted.

THEOREM 19b. If

$$
0<\mu_{1} \leqq \mu_{2} \leqq \mu_{3} \leqq \cdots, \lim _{k \rightarrow \infty} \mu_{k}=\infty,
$$

are the eigen values of $R^{\wedge}$ then for each $k=1,2, \ldots$

$$
\lambda_{n k}=t\left(x_{0}\right)-n^{-\omega} L\left(n^{-1}\right)\left[\mu_{k}+o(1)\right]
$$

as $n \rightarrow \infty$.

Let us consider as an example the case where $t(x)$ has a unique absolute maximum at $x_{0}$ and is twice continuously differentiable in a neighborhood of $x_{0}$. Then $t^{\prime}\left(x_{0}\right)=0$. We assume that $t^{\prime \prime}\left(x_{0}\right)=-\sigma^{2}<0$. Then in terms of the notation of $\S 15, \omega=2, \sigma_{1}=\sigma_{2}=\sigma^{2} / 2, L \equiv 1$. Consequently

$$
s(u)=\pi \sigma^{2}\left(\sin ^{2} \xi_{0}\right) u^{2} \quad-\infty<u<\infty,
$$

and the eigen values $0<\mu_{1} \leqq \cdots$ of $R^{\wedge}$ are easily seen to be the eigen values of the differential operator $R=\psi^{-1} R^{\wedge} \psi$ defined by

$$
R f \cdot(z)=-\frac{\sigma^{2}}{4} \sin ^{2} \xi_{0} f^{\prime \prime}(z),
$$

the domain $\boldsymbol{D}(R)$ consisting of those functions $f(z)$ with support in $-1 \leqq z \leqq 1$ which are such that $f(z)$ and $f^{\prime}(z)$ are absolutely continuous for $-1<z<1, f^{\prime \prime}(z) \in L^{2}(-1,1)$ and $f(1-)=f(-1+)=0$. Since $\mu_{k}=\sigma^{2}\left(\sin \xi_{0}\right)^{2} k^{2} / 8$ we find that

$$
\lambda_{n, k}=t\left(x_{0}\right)-\sigma^{2}\left(1-x_{0}^{2}\right) k^{2} / 8 n^{2}+o\left(n^{-2}\right)
$$

as $n \rightarrow \infty$ for each $k=1,2, \cdots$. See $(6)$ of $\S 1$.

\section{APPENDIX}

The Jacobi polynomials $P_{n}^{(\alpha, \beta)}(x)$ defined in $\S 1$ satisfy the recursion 
formula, [1; p. 168],

$$
x P_{n}^{(\alpha, \beta)}(x)=A_{n} P_{n+1}^{(\alpha, \beta)}(x)+B_{n} P_{n}^{(\alpha \beta \beta)}(x)+C_{n} P_{n-1}^{(\alpha, \beta)}(x)
$$

where

$$
\begin{gathered}
A_{n}=2(n+1)(n+\alpha+\beta+1)(2 n+\alpha+\beta+1)^{-1} \\
\cdot(2 n+\alpha+\beta+2)^{-1}, \\
B_{n}=-\left(\alpha^{2}-\beta^{2}\right)(2 n+\alpha+\beta+2)^{-1}(2 n+\alpha+\beta)^{-1}, \\
C_{n}=2(n+\alpha)(n+\beta)(2 n+\alpha+\beta)^{-1}(2 n+\alpha+\beta+1)^{-1} .
\end{gathered}
$$

We have the following limit relation

$$
\lim _{n \rightarrow \infty} n^{-\alpha} P_{n}^{(\alpha, \beta)}\left(\cos z n^{-1}\right)=(z / 2)^{-\alpha} J_{\alpha}(z)
$$

uniformly for $z$ in any bounded subset of the complex $z$ plane, [1; p.173]. We also have

$$
h_{n}^{1 / 2} P_{n}^{(\alpha, \beta)}(\cos \theta)\left[w_{\alpha, \beta}(\cos \theta)\right]^{1 / 2} \sin ^{1 / 2} \theta-\sqrt{\frac{2}{n}} \cos (N \theta+\gamma) \rightarrow 0
$$

as $n \rightarrow \infty$ uniformly for $\varepsilon \leqq \theta \leqq \pi-\varepsilon$, if $\varepsilon>0$. Here

$$
N=n+(\alpha+\beta+1) / 2, \quad \gamma=-\left(\alpha+\frac{1}{2}\right) \pi / 2 .
$$

See $[12 ;$ p. 190].

Let $q=\max (\alpha, \beta,-1 / 2)$; then

$$
\left|P_{n}^{(\alpha, \beta)}(\cos u)\right| \leqq A(n+1)^{q} \quad-\infty<u<\infty
$$

where $A$ depends upon $\alpha$ and $\beta,[12$, p. 163]. Furthermore if $w=$ $u+i v$ then, see [12; p. 190],

$$
\left|P_{n}^{(\alpha, \beta)}(\cos w)\right| \leqq A(n+1)^{-1 / 2} e^{|v| n}
$$

uniformly for $|v| \geqq v_{0}>0$. Here $A$ depends only upon $\alpha, \beta$ and $v_{0}$. Applying Hadamard's three lines theorem to $P_{n}^{(\alpha, \beta)}(\cos w)$ we find that for all $w$

$$
\left|P_{n}^{(\alpha, \beta)}(\cos w)\right| \leqq A(n+1)^{q} e^{|v| n}
$$

where $A$ and $q$ are independent of $n$ and $w$. The inequality (8), although crude, has the advantage that it holds uniformly in $n$ and $w$.

We set

$$
Q_{n}(z)=\frac{1}{2} \int_{-1}^{1} P_{n}^{(\alpha, \beta)}(t)(z-t)^{-1} w_{\alpha, \beta}(t) d t
$$


for all complex $z \notin[-1,1]$. We then have

$$
Q_{n}(z)=(1-z)^{\alpha}(z+1)^{\beta} Q_{n}^{(\alpha, \beta)}(z),
$$

where $Q_{n}^{(\alpha, \beta)}(z)$ is the standard Jacobi function of the second kind, $\left[1 ;\right.$ p. 170]. We will use $Q_{n}(z)$ rather than $Q_{n}^{(\alpha, \beta)}(z)$ because it is single valued in the $z$-plane slit from -1 to 1 . If we set

$$
\begin{aligned}
& Q_{n}^{+}(x)=\lim _{\varepsilon \rightarrow 0+} Q_{n}(x+i \varepsilon) \\
& Q_{n}^{-}(x)=\lim _{\varepsilon \rightarrow 0+} Q_{n}(x-i \varepsilon)
\end{aligned}
$$

then for $-1<x<1$

$$
Q_{n}^{-}(x)-Q_{n}^{+}(x)=\pi i P_{n}^{(\alpha, \beta)}(x) w_{\alpha, \beta}(x) .
$$

By an argument analogous to that used to prove (8), see [12; p. 219], we can show that if $v=I_{m} w \neq 0$ then

$$
\left|Q_{n}(\cos w) \sin ^{2} w\right| \leqq A(n+1)^{q} e^{-|v|(n-2)}
$$

where $A$ and $q$ are independent of $n$ and $w$. Like (8) this inequality is quite crude, but it is important because it is uniform in $n$ and $w$.

\section{BIBLIOGRAPHY}

1. A. Erdélyi et al., Higher transcendental functions vol. 2, New York 1953.

2. U. Grenander and G. Szegö, Toeplitz forms and their applications, Berkeley and Los Angeles 1958.

3. D. Guy, Hankel multiplier transformations and weighted p-norms, Trans. Amer. Math. Soc., 95 (1960), 137-189.

4. I. I. Hirschman, Jr., Variation diminishing Hankel transforms, Jour. d Anal. Math., 8 (1960-61), 307-336.

5. - Extreme eigen values of Toeplitz forms associated with orthogonal polynomials, to appear in Jour. d Anal. Math.

6. M. Kac, W. L. Murdock and G. Szegö, On the eigen values of certain Hermitian forms, J. Rat. Mech. Anal., 2 (1953), 767-800.

7. T. Kato, Quadratic Forms in Hilbert spaces and asymptotic perturbation series, Mimeographed Notes, Berkeley 1955.

8. R. Nevanlinna, Eindeutige analytische functionen Berlin 1936.

9. S. V. Parter, Extreme eigenvalues of Toeplitz forms and applications to elliptic difference equations, Trans. Amer. Math. Soc., 99 (1961), 153-193.

10. - On the extreme eigenvalues of truncated Toepiitz matrices, Bull. Amer. Math. Soc., 67 (1961), 191-196.

11. On the extreme eigenvalues of Toeplitz matrices, Trans. Amer. Math. Soc., 100 (1961), 263-276.

12. G. Szegö, Orthogonal Polynomials, Amer. Math. Soc. Col. Pub., 23, New York, 1939.

13. B. von Szegy-Nagy, Spectraldarstellung Linearer Transformationen des Hilbertschen Raumes, Berlin 1942.

14. H. Widom, On the eigenvalues of certain Hermitian operators, Trans. Amer. Math. 
Soc., 88 (1958), 491-522.

15. H. Widom, Stable procesess and integral equations, Trans. Amer. Math. Soc., 98 (1961), 430-439.

16. - Extreme eigenvalues of translation kernels, Trans. Amer. Math. Soc., 100 (1961), 252-262.

17. - Extreme eigenvalues of $N$-dimensional convolution operators, Trans. Amer. Math. Soc., 106 (1963), 391-414. 



\section{PACIFIC JOURNAL OF MATHEMATICS}

\section{EDITORS}

Robert Osserman

Stanford University

Stanford, California

M. G. Arsove

University of Washington

Seattle 5 , Washington
J. DugundjI

University of Southern Califorma: Los Angeles 7, California

Lowell J. Paige

University of California

Los Angeles 24, California

\section{ASSOCIATE EDITORS}

E. F. BECKENBACH

B. H. NeumanN

F. WOLF

K. YosIDA

\section{SUPPORTING INSTITUTIONS}

UNIVERSITY OF BRITISH COLUMBIA

CALIFORNIA INSTITUTE OF TECHNOLOGY

UNIVERSITY OF CALIFORNIA

MONTANA STATE UNIVERSITY

UNIVERSITY OF NEVADA

NEW MEXICO STATE UNIVERSITY

OREGON STATE UNIVERSITY

UNIVERSITY OF OREGON

OSAKA UNIVERSITY

UNIVERSITY OF SOUTHERN CALIFORNIA
STANFORD UNIVERSITY

UNIVERSITY OF TOKYO

UNIVERSITY OF UTAH

WASHINGTON STATE UNIVERSITY

UNIVERSITY OF WASHINGTON

AMERICAN MATHEMATICAL SOCIETY CALIFORNIA RESEARCH CORPORATION SPACE TECHNOLOGY LABORATORIES NAVAL ORDNANCE TEST STATION 


\section{Pacific Journal of Mathematics}

\section{Vol. 14, No. 1 \\ May, 1964}

Richard Arens, Normal form for a Pfaffian .........................

Charles Vernon Coffman, Non-linear differential equations on cones in Banach

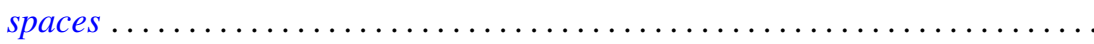

Ralph DeMarr, Order convergence in linear topological spaces ..............

Peter Larkin Duren, On the spectrum of a Toeplitz operator ................

Robert E. Edwards, Endomorphisms of function-spaces which leave stable all

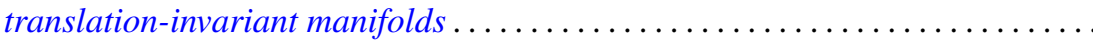

Erik Maurice Ellentuck, Infinite products of isols . . . . . . . . . . . . . . . . 49

William James Firey, Some applications of means of convex bodies . . . . . . . . 53

Haim Gaifman, Concerning measures on Boolean algebras ............. 61

Richard Carl Gilbert, Extremal spectral functions of a symmetric operator. . . . . . 75

Ronald Lewis Graham, On finite sums of reciprocals of distinct nth powers ..... 85

Hwa Suk Hahn, On the relative growth of differences of partition functions ...... 93

Isidore Isaac Hirschman, Jr., Extreme eigen values of Toeplitz forms associated

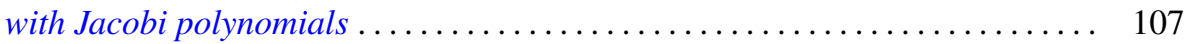

Chen-jung Hsu, Remarks on certain almost product spaces . . . . . . . . . . . 163

George Seth Innis, Jr., Some reproducing kernels for the unit disk . . . . . . . . . 177

Ronald Jacobowitz, Multiplicativity of the local Hilbert symbol . . . . . . . . . . . 187

Paul Joseph Kelly, On some mappings related to graphs ................. 191

William A. Kirk, On curvature of a metric space at a point . . . . . . . . . . . . 195

G. J. Kurowski, On the convergence of semi-discrete analytic functions . . . . . . . 199

Richard George Laatsch, Extensions of subadditive functions . . . . . . . . . . . 209

V. Marić, On some properties of solutions of $\Delta \psi+A\left(r^{2}\right) X \nabla \psi+C\left(r^{2}\right) \psi=0 \ldots 217$

William H. Mills, Polynomials with minimal value sets . . . . . . . . . . . 225

George James Minty, Jr., On the monotonicity of the gradient of a convex

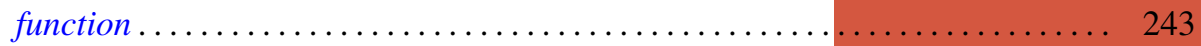

George James Minty, Jr., On the solvability of nonlinear functional equations of 'monotonic' type ................................... 249

J. B. Muskat, On the solvability of $x^{e} \equiv e(\bmod p) \ldots \ldots \ldots \ldots \ldots \ldots \ldots \ldots . \ldots \ldots$

Zeev Nehari, On an inequality of $P . R$. Bessack ................... 261

Raymond Moos Redheffer and Ernst Gabor Straus, Degenerate elliptic

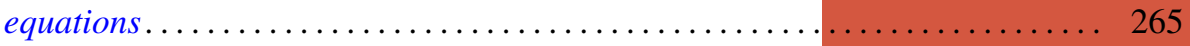

Abraham Robinson, On generalized limits and linear functionals . . . . . . . . . 269

Bernard W. Roos, On a class of singular second order differential equations with a

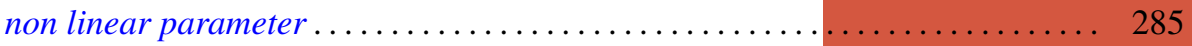

Tôru Saitô, Ordered completely regular semigroups . . . . . . . . . . . . . . . . 295

Edward Silverman, A problem of least area ....................... 309

Robert C. Sine, Spectral decomposition of a class of operators . . . . . . . . . 333

Jonathan Dean Swift, Chains and graphs of Ostrom planes . . . . . . . . . . . 353

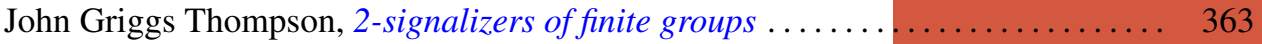

Harold Widom, On the spectrum of a Toeplitz operator . . . . . . . . . . . . . 365 